\begin{tabular}{|c|l|}
\hline Title & Molecular mechanisms of the photostability of indigo \\
\hline Author(s) & Yamazaki, Shohei; Sobolewski, A ndrzej L.; Domcke, Wolfgang \\
\hline Citation & $\begin{array}{l}\text { Physical Chemistry Chemical Physics, 13(4), 1618-1628 } \\
\text { https://doi.org/10.1039/c0cp01901a }\end{array}$ \\
\hline Issue Date & 2011-01-28 \\
\hline Doc URL & http://hdl.handle.net/2115/47361 \\
\hline Rights & Phys. Chem. Chem. Phys., 2011, 13, 1618_1628- Reproduced by permission of the PCCP Owner Societies \\
\hline Type & article (author version) \\
\hline File Information & PCcP13_4_1618_1628.pdf \\
\hline
\end{tabular}

Instructions for use 


\title{
Molecular mechanisms of the photostability of indigo $\dagger$
}

\author{
Shohei Yamazaki, ${ }^{* a b}$ Andrzej L. Sobolewski ${ }^{c}$ and Wolfgang Domcke ${ }^{b}$ \\ ${ }^{a}$ Division of Chemistry, Graduate School of Science, Hokkaido University, Sapporo 060-0810, Japan. \\ E-mail:shohei@mail.sci.hokudai.ac.jp \\ ${ }^{b}$ Department of Chemistry, Technical University of Munich, D-85747 Garching, Germany \\ ${ }^{c}$ Institute of Physics, Polish Academy of Sciences, PL-02668 Warsaw, Poland
}

$\dagger$ Electronic supplementary information (ESI) available: Cartesian coordinates of $\mathbf{1}$ and $\mathbf{2}$ at minimum and conical-intersection geometries, molecular orbitals of $\mathbf{2}$ at conical intersections and bond lengths of 2 at minimum and conical intersection. 


\begin{abstract}
The photophysics of indigo as well as of bispyrroleindigo, the basic chromophore of indigo, has been investigated with ab initio electronic-structure calculations. Vertical electronic excitation energies and excited-state potential-energy profiles have been calculated with the CASSCF, CASPT2 and CC2 methods. The calculations reveal that indigo and bispyrroleindigo undergo intramolecular single-proton transfer between adjacent $\mathrm{N}-\mathrm{H}$ and $\mathrm{C}=\mathrm{O}$ groups in the ${ }^{1} \pi \pi^{*}$ excited state. The nearly barrierless proton transfer provides the pathway for a very efficient deactivation of the ${ }^{1} \pi \pi^{*}$ state via a conical intersection with the ground state. While a low-lying $\mathrm{S}_{1}-\mathrm{S}_{0}$ conical intersection exists also after double-proton transfer, the latter reaction path exhibits a much higher barrier. The reaction path for trans $\rightarrow$ cis photoisomerization via the twisting of the central $\mathrm{C}=\mathrm{C}$ bond has been investigated for bispyrroleindigo. It has been found that the twisting of the central $\mathrm{C}=\mathrm{C}$ bond is unlikely to play a role in the photochemistry of indigo, because of a large potential-energy barrier and a rather high energy of the $\mathrm{S}_{1-}$ $\mathrm{S}_{0}$ conical intersection of twisted structure. These findings indicate that the exceptional photostability of indigo is the result of rapid internal conversion via intramolecular single-proton transfer, combined with the absence of a low-barrier reaction path for the generation of the cis isomer via trans $\rightarrow$ cis photoisomerization.
\end{abstract}




\section{Introduction}

Indigo (1, see Scheme 1) is one of the most ancient and most widely used natural dyes. ${ }^{1-4}$ Indigo is highly photostable. Numerous spectroscopic studies have been performed over several decades to elucidate the basic mechanisms of the photochemistry of indigo.

One characteristic feature of the photochemistry of indigo is the absence of a photoinduced trans $\rightarrow$ cis isomerization, that is, twisting of the central $\mathrm{C}=\mathrm{C}$ bond. Absorption spectrum suggests that the trans structure of indigo is retained after photoexcitation and internal conversion. ${ }^{5,6}$ The same applies for ringsubstituted derivatives of indigo, which possess intramolecular hydrogen bonds between the adjacent $\mathrm{C}=\mathrm{O}$ and $\mathrm{N}-\mathrm{H}$ groups. ${ }^{5,6}$ For indigo derivatives in which the intramolecular hydrogen bonds are missing, on the other hand, the cis isomer is readily generated by photoexcitation. ${ }^{5-14}$ For these indigo derivatives, it has been proposed that triplet states are involved as intermediates in the trans $\rightarrow$ cis photoisomerization process. ${ }^{15-22}$ Indigo itself, however, exhibits a very low quantum yield of intersystem crossing. ${ }^{23-25}$

Another experimental observation related to the photostability of indigo is the extremely short lifetime of the lowest excited singlet state. It has been found that the $\mathrm{S}_{1}$ lifetimes of indigo as well as of its ringsubstituted compounds, such as indigo carmine (5,5'-indigodisulfonic acid disodium salt) or Tyrian purple (6,6'-dibromoindigo), are of the order of several tens or hundreds of picoseconds in the natural keto form. ${ }^{23,24,26-30}$ The excited-state lifetime of indigo is much shorter than the $\mathrm{S}_{1}$ lifetimes of the leuco (reduced) form of indigo, ${ }^{23,31}$ dehydroindigo (the oxidized form of indigo), ${ }^{32}$ as well as other derivatives, such as thioindigo, ${ }^{13,20,27,33}$ which are of the order of nanoseconds. These findings suggest that particularly efficient radiationless decay mechanisms exist in indigo which are responsible for its exceptional photostability.

Excited-state intramolecular proton transfer (ESIPT) along the hydrogen bonds between the adjacent $\mathrm{C}=\mathrm{O}$ and $\mathrm{N}-\mathrm{H}$ groups has been considered as a possible mechanism of the efficient deactivation of indigo. ${ }^{34,35}$ Kobayashi and Rentzepis attributed the fast decay of the transient absorption of indigo to 
ultrafast internal conversion which is enhanced by excited-state proton transfer rather than twisting of the central $\mathrm{C}=\mathrm{C}$ bond. ${ }^{26}$ Seixas de Melo et al. found that the fluorescence of indigo and of derivatives possessing at least one intramolecular hydrogen bond exhibits a biexponential decay. ${ }^{24,30}$ They assigned the short-time and long-time components to decays of the reactant (keto) and the product (enol) forms of ESIPT, respectively. Very recently, Iwakura et al. detected the transient formation of a single $\mathrm{OH}$ bond (rather than of two $\mathrm{OH}$ bonds) in the excited state of indigo carmine by a pump-probe measurement with sub-5-femtosecond time resolution. ${ }^{36,37}$ They concluded that the ESIPT in indigo takes place via a single-proton transfer (SPT) process rather than a double-proton transfer (DPT) process. Elsaesser et al., on the other hand, could not find any evidence of ESIPT in indigo on the basis of transient infrared spectra. ${ }^{28}$ Nagasawa et al. found that the $\mathrm{S}_{1}$ lifetime of indigo carmine in solution strongly depends on solvent polarity. ${ }^{29}$ They concluded that intermolecular hydrogen bonds between solute and solvent molecules play a more essential role in the deactivation process of indigo than the intramolecular hydrogen bonds of the solute molecule. To clarify unambiguously the role of ESIPT for the photostability of indigo, computational studies of the competing photochemical reaction paths are required.

Most computational studies of the excited states of indigo have focused on the remarkably long wavelength of absorption, which is the source of the deep blue color of indigo dyes. Semiempirical calculations performed in the 1960 s to the 1980 s led to the conclusion that the basic chromophore of the indigo dyes is the central $\mathrm{C}=\mathrm{C}$ bond together with the adjacent $\mathrm{C}=\mathrm{O}$ and $\mathrm{N}-\mathrm{H}$ groups. ${ }^{38-43}$ The excitation energies of singlet excited states of indigo have been extensively studied with ab initio methods ${ }^{44,45}$ as well as, more recently, with time-dependent density functional theory (TDDFT) and with inclusion of solvation effects by a polarizable continuum model ${ }^{46-49}$ However, little attention has been paid to the photochemical reactions of indigo, such as ESIPT and trans $\rightarrow$ cis photoisomerization. To our knowledge, only a few computational studies were carried out with semiempirical methods to examine the photoinduced reactions of indigo dyes. ${ }^{50-52}$ 
In the present work, the photoinduced reactions of indigo were studied using ab initio methods. The complete-active-space self-consistent-field (CASSCF), second-order perturbation theory based on the CASSCF reference (CASPT2) $)^{53}$ and the approximate second-order coupled-cluster singles-and-doubles $(\mathrm{CC} 2)^{54}$ methods were employed. We studied indigo itself (1, see Scheme 1) as well as the truncated compound bispyrroleindigo (2, see Scheme 1), which represents the basic chromophore of indigo. Our goal is to elucidate with first-principles calculations the mechanisms which are responsible for the photostability of indigo at the molecular level. To this end, the potential-energy (PE) profiles of singleand double-proton-transfer reactions in the photoexcited state as well as of trans $\rightarrow$ cis photoisomerization were calculated.

In particular, we focus on conical intersections $(\mathrm{CIs})^{55}$ which are relevant for the photoinduced reactions of $\mathbf{1}$ and 2. CIs are crossing seams of the PE surfaces of different electronic states, which are exactly degenerate at these seams. Owing to extremely large nonadiabatic couplings at CIs, the latter are particularly efficient funnels for the radiationless decay of photoexcited molecules to the electronic ground state. ${ }^{55}$ It has recently been shown for several generic ESIPT systems that the photochemistry is likely to be dominated by ultrafast deactivation through CIs of the $\mathrm{S}_{1}$ and $\mathrm{S}_{0}$ states which are accessed by ESIPT, in combination with out-of-plane torsion. ${ }^{56-60}$ Photoisomerization and internal conversion through $\mathrm{CIs}$ which are reached by the twisting of $\mathrm{C}=\mathrm{C}$ double bonds are another widely accepted paradigm for ultrafast radiationless decay in unsaturated hydrocarbons. ${ }^{61-63}$ The accessibility of CIs associated with competing processes in the excited states of indigo is explored in the present work, with the goal of providing detailed insights into the origin of the photostability of indigo.

\section{Computational methods}

The ground-state equilibrium geometries of $\mathbf{1}$ and $\mathbf{2}$ were determined with the second-order MøllerPlesset (MP2) method. The excited-state minimum-energy geometries along selected reaction pathways were optimized with the CASSCF method. The geometries of CIs between the ${ }^{1} \pi \pi^{*}$ excited state and the 
$\mathrm{S}_{0}$ state $\left({ }^{1} \pi \pi^{*}-\mathrm{S}_{0}\right.$ CIs) were also determined with the CASSCF method. Single-point internally contracted CASPT2 calculations ${ }^{53}$ were performed at the MP2-optimized geometries of the ground state as well as at the CASSCF-optimized geometries of the excited-state reaction paths and the ${ }^{1} \pi \pi^{*}-\mathrm{S}_{0}$ CIs. The CC2 method ${ }^{54}$ was used for the calculations of the vertical excitation energies at the $\mathrm{S}_{0}$ equilibrium geometries as well as for a part of the calculations of the ${ }^{1} \pi \pi^{*}$ PE surface. A level shift parameter of 0.3 was employed in the CASPT2 calculations. ${ }^{64}$ The resolution-of-the-identity (RI) approximation was applied in the MP2 and CC2 calculations. ${ }^{65,66}$ The correlation-consistent polarized valence double- $\zeta$ (ccpVDZ) basis set ${ }^{67}$ was employed for all calculations. The program package MOLPRO ${ }^{68}$ was used for the CASSCF and CASPT2 calculations, while the package TURBOMOLE ${ }^{69}$ was used for the MP2 and CC2 calculations.

The minimum-energy reaction paths in the excited states were determined as relaxed scans along the reaction coordinates for ESIPT (SPT and DPT) of $\mathbf{1}$ and $\mathbf{2}$, as well as for the trans $\rightarrow$ cis photoisomerization of $\mathbf{2}$, see the overview in Fig. 1. The photoisomerization of $\mathbf{1}$ was not studied, because the computing time would have been excessive. For each pathway, the value of one (or two) reaction coordinate(s) were fixed, while the excited-state energy was optimized with respect to all other internal coordinates. The reaction coordinates appropriate for the ESIPT process are the differences of the lengths of adjacent $\mathrm{NH}$ and $\mathrm{OH}$ bonds (see Scheme 1 for the numbering of atoms)

$$
s_{a}=\frac{1}{2}\left[r\left(N_{1 a} H_{7 a}\right)-r\left(O_{6 b} H_{7 a}\right)\right]
$$

and

$$
s_{b}=\frac{1}{2}\left[r\left(N_{1 b} H_{7 b}\right)-r\left(O_{6 a} H_{7 b}\right)\right]
$$

which represent the proton transfer along the two intramolecular hydrogen bonds, respectively. For SPT, $s_{a}$ (or $s_{b}$ ) was fixed, optimizing $s_{b}$ (or $s_{a}$ ) as well as the other internal coordinates in $C_{s}$ symmetry. For DPT, the geometry optimizations were carried out in $C_{2 h}$ symmetry, where $s_{a}=s_{b}$ and the reaction coordinate is just referred to as $s$. 
The reaction coordinate $\varphi$ for the trans $\rightarrow$ cis photoisomerization is defined by the dihedral angles representing the twisting of the central $\mathrm{C}=\mathrm{C}$ bond as

$$
\varphi=\frac{1}{2}\left[\delta\left(N_{1 a} C_{2 a} C_{2 b} C_{3 b}\right)+\delta\left(N_{1 b} C_{2 b} C_{2 a} C_{3 a}\right)\right]
$$

No symmetry constraint was employed in the optimization of the twisting path. The geometries of the ${ }^{1} \pi \pi^{*}-\mathrm{S}_{0}$ CIs were optimized for the SPT and DPT structures with $C_{s}$ symmetry constraint, and for C=C twisted structures without symmetry constraints $\left(C_{l}\right.$ symmetry).

Various active spaces were applied in the CASSCF calculations for $\mathbf{1}$ and $\mathbf{2}$. We denote active spaces as $(M, N / k \pi l \mathrm{n})$, where $M$ and $N$ are the numbers of active electrons and orbitals, respectively. $k$ and $l$ define the number of $\pi$ and $\mathrm{n}$ (in-plane lone pair) orbitals in the active space, respectively $(k+l=N)$. The active spaces which include only $\pi$ orbitals are denoted as $(M, N / k \pi)$. While $\mathbf{1}$ has 22 electrons in 20 $\pi$ orbitals in total, not all of them could be included in the active space. For the ${ }^{1} \pi \pi^{*}$ state of $\mathbf{1}$, we applied the $(10,10 / 10 \pi)$ active space for the CASSCF geometry optimizations and the CASPT2 singlepoint calculations of ESIPT pathways, in which the energies of the lowest two singlet states $\left(\mathrm{S}_{0}\right.$ and $\left.{ }^{1} \pi \pi^{*}\right)$ were averaged with equal weights. For CASPT2 single-point calculations of vertical excitation energies of $\mathbf{1}$, the $(14,14 / 14 \pi)$ active space was employed. For the ${ }^{1} \pi \pi^{*}$ state of 2 , we used the $(14,12 / 12 \pi)$ active space, which includes all $\pi$ orbitals of this molecule. For the trans $\rightarrow$ cis photoisomerization of $\mathbf{2}$, we optimized the minimum-energy geometries in the ${ }^{1} \mathrm{n} \pi^{*}$ state as well as in the ${ }^{1} \pi \pi^{*}$ state as a function of the twisting coordinate $\varphi$. In the optimization of the geometry of the ${ }^{1} n \pi^{*}$ state, the $(12,11 / 10 \pi 1 \mathrm{n})$ active space was employed, which includes the lone pair orbital of one $\mathrm{O}$ atom, and the $\mathrm{S}_{0}$ and ${ }^{1} \mathrm{n} \pi^{*}$ states were averaged with equal weights. CASPT2 energies along twisting pathways optimized in the ${ }^{1} \pi \pi^{*}$ and ${ }^{1} \mathrm{n} \pi^{*}$ states were calculated with the $(14,12 / 10 \pi 2 n)$ active space. In this case, the energies of the lowest five singlet states, i.e. $\mathrm{S}_{0}$, two ${ }^{1} \pi \pi^{*}$ and two ${ }^{1} \mathrm{n} \pi^{*}$ states, were averaged. The $(14,12 / 12 \pi)$ and $(14,12 / 10 \pi 2 n)$ active spaces were used for the CASPT2 calculations of the vertical excitation energies of 2 . 


\section{Results and discussion}

\subsection{Vertical excitation energies}

For both 1 and 2, geometry optimizations of the $\mathrm{S}_{0}$ state with the MP2 method resulted in structures of $C_{2 h}$ symmetry. The Cartesian coordinates of the optimized geometries are given in the ESI. $\dagger$ At the respective $\mathrm{S}_{0}$ equilibrium geometries, we have calculated the vertical excitation energies with the CC2 method for the lowest excited singlet state of each irreducible representation of the $C_{2 h}$ point group, i.e., $2^{1} A_{g}, 1^{1} A_{u}, 1^{1} B_{g}$ and $1^{1} B_{u}\left(1^{1} A_{g}\right.$ is the $\mathrm{S}_{0}$ state). The results are given in Tables 1 and 2 . The lowest excited state of 1 is $1^{1} B_{u}$, which is of ${ }^{1} \pi \pi^{*}$ character and is responsible for the absorption in the visible region $(2.34 \mathrm{eV}$, corresponding to $530 \mathrm{~nm})$ with a large oscillator strength $(0.375)$, see Table 1 . The $1^{1} B_{u}\left(\pi \pi^{*}\right)$ excitation energy obtained with the CC2 method is in good agreement with the absorption spectrum of indigo in the gas phase ${ }^{40,70-72}$ as well as with the results of TDDFT calculations. ${ }^{46-48}$ This state clearly is the initially populated state in the photoabsorption of indigo. For $\mathbf{2}$, the excitation energy of the $1^{1} B_{u}\left(\pi \pi^{*}\right)$ state obtained at the CC2 level $(2.68 \mathrm{eV})$ is in agreement with the absorption spectrum of a tetramethylated derivative of $2,^{40}$ see Table 2 .

The $1^{1} B_{u}\left(\pi \pi^{*}\right)$ state is essentially a single excitation from the highest occupied molecular orbital (HOMO) to the lowest unoccupied molecular orbital (LUMO). Fig. 2 shows the HOMOs and LUMOs of 1 and 2, obtained by a restricted Hartree-Fock (RHF) calculation. The RHF wave function is the reference function for the $\mathrm{CC} 2$ calculations. The HOMO mainly consists of the $\pi$ orbital on the central $\mathrm{C}=\mathrm{C}$ bond and the $\mathrm{p}_{\mathrm{z}}$ orbitals on the $\mathrm{N}$ atoms, while the LUMO is dominated by the central $\pi^{*}$ orbital and the $\mathrm{p}_{z}$ orbitals on the $\mathrm{O}$ atoms. Inspection of Fig. 2 shows that the HOMOs and the LUMOs of 1 and 2 are very similar: the orbitals are localized on the five-membered rings and on the central $\mathrm{C}=\mathrm{C}$ bond.

The higher excited states $2^{1} A_{g}, 1^{1} A_{u}$ and $1^{1} B_{g}$ are of ${ }^{1} \pi \pi^{*},{ }^{1} \mathrm{n} \pi^{*}$ and ${ }^{1} \mathrm{n} \pi^{*}$ character, respectively, for $\mathbf{1}$ as well as $\mathbf{2}$. The small values of the oscillator strengths of these states (see Tables 1 and 2) confirm that 
they are dark states in absorption from the $\mathrm{S}_{0}$ state. The oscillator strengths of the $2^{1} A_{g}\left(\pi \pi^{*}\right)$ and $1^{1} B_{g}\left(\mathrm{n} \pi^{*}\right)$ states are strictly zero in $C_{2 h}$ symmetry. The CC2 results indicate that the ${ }^{1} \pi \pi^{*}\left(1^{1} B_{u}\right.$ and $\left.2^{1} A_{g}\right)$ excitation energies of $\mathbf{2}$ are higher than those of $\mathbf{1}$, while the ${ }^{1} \mathrm{n} \pi^{*}\left(1^{1} A_{u}\right.$ and $\left.1^{1} B_{g}\right)$ energies of $\mathbf{2}$ are lower than those of 1 . For the $1^{1} B_{u}\left(\pi \pi^{*}\right)$ state this tendency is in agreement with experiment, see Tables 1 and 2.

The energy of another excited state, corresponding to double excitation from the HOMO to the LUMO of Fig. 2, has been calculated using the RHF wave function for the doubly excited configuration, denoted as $\left(\pi^{*}\right)^{2}$, as the reference for the CC2 calculation. The resulting excitation energies of $\mathbf{1}$ and $\mathbf{2}$, given in the last lines of Tables 1 and 2 , are close to the respective energies of the $1^{1} B_{u}\left(\pi \pi^{*}\right)$ state, although the doubly excited state is optically forbidden, being of ${ }^{1} A_{g}$ symmetry.

Vertical excitation energies of $\mathbf{1}$ and $\mathbf{2}$ have also been calculated with the CASPT2 method. For $\mathbf{1}$, the CASPT2 calculation with the $(14,14 / 14 \pi)$ active space has resulted in an excitation energy of $1.81 \mathrm{eV}$, which is close to an early CASPT2 result $(1.96 \mathrm{eV})$ reported by Serrano-Andrés and Roos, ${ }^{45}$ using the $(14,12 / 12 \pi)$ active space. This excitation energy is significantly lower than the experimental result of about $2.30 \mathrm{eV}$. It seems that the CASSCF active spaces which are currently computationally feasible are too small for the prediction of accurate excitation energies of 1 with the CASPT2 method. For the $1^{1} B_{u}\left(\pi \pi^{*}\right)$ state of $\mathbf{2}$, the CASPT2 excitation energy is in better agreement with the CC2 energy and the experimental value, see Table 2 . The CASPT2 results for the ${ }^{1} \pi \pi^{*}\left(1^{1} B_{u}\right.$ and $\left.2^{1} A_{g}\right)$ states as well as the ${ }^{1} \mathrm{n} \pi^{*}\left(1^{1} A_{u}\right.$ and $\left.1{ }^{1} B_{g}\right)$ states are all lower than the corresponding CC2 excitation energies. The CASPT2 method also predicts that the $1^{1} B_{u}\left(\pi \pi^{*}\right)$ energy of $\mathbf{2}$ is higher than that of $\mathbf{1}$, in agreement with experiment.

\subsection{Conical intersections}

Fig. 3 shows the geometries of the ${ }^{1} \pi \pi^{*}-\mathrm{S}_{0}$ CIs of $\mathbf{1}$ and $\mathbf{2}$, optimized with the CASSCF method, as well as their energies calculated with the CASPT2 method. The Cartesian coordinates of the CI 
geometries are given in the ESI. $\dagger$ The $(10,10 / 10 \pi)$ and $(14,12 / 12 \pi)$ active spaces were used in the calculations of $\mathbf{1}$ and $\mathbf{2}$, respectively. The CASPT2 energies given in Fig. 3 are the average of the $\mathrm{S}_{0}$ and $\mathrm{S}_{1}$ energies. The difference of the $\mathrm{S}_{0}$ and $\mathrm{S}_{1}$ CASPT2 energies, which was always less than $0.4 \mathrm{eV}$, is due to different dynamical electron correlation, which lifts the degeneracy of the $\mathrm{S}_{0}$ and $\mathrm{S}_{1}$ states obtained at the CASSCF level. Although not perfect, the average value of the CASPT2 energies has been found to be a useful approximation for the determination of the relative energies of CIs in large molecules, for which geometry optimizations at the multi-reference configuration interaction (MRCI) level are not feasible.

We have found three types of ${ }^{1} \pi \pi^{*}-\mathrm{S}_{0}$ CIs in $\mathbf{1}$ and $\mathbf{2}$, see Fig. 3. The first corresponds to a SPT structure $\left(\mathrm{CI}_{\mathrm{SPT}}\right.$ in Fig. 3a): the proton of one $\mathrm{NH}$ bond is transferred to the adjacent $\mathrm{O}$ atom, resulting in the mono-enol form. The second corresponds to a DPT structure $\left(\mathrm{CI}_{\mathrm{DPT}}\right.$ in Fig. $\left.3 \mathrm{~b}\right)$ : the protons of both NH bonds are transferred, resulting in the di-enol form. The third type corresponds to a twisted structure $\left(\mathrm{CI}_{\text {twist }}\right.$ in Fig. $\left.3 \mathrm{c}\right)$ : the central $\mathrm{C}=\mathrm{C}$ bond exhibits a large twist angle (nearly $\left.90^{\circ}\right) . \mathrm{CI}_{\mathrm{SPT}}$ and $\mathrm{CI}_{\mathrm{DPT}}$ are reached by ESIPT processes (Figs. 1a and $1 \mathrm{~b}$ ), while $\mathrm{CI}_{\text {twist }}$ may be accessed during trans $\rightarrow$ cis photoisomerization (Fig. 1c). The $\mathrm{CI}_{\text {twist }}$ of $\mathbf{1}$ could not be determined with the available computational resources.

As shown in Fig. 3, the CASPT2 energies of $\mathrm{CI}_{\mathrm{SPT}}$ and $\mathrm{CI}_{\mathrm{DPT}}$ of 2 are $1.80 \mathrm{eV}$ and $2.22 \mathrm{eV}$, respectively, relative to the $S_{0}$ equilibrium geometry. They are lower than the vertical excitation energy of the $1^{1} B_{u}\left(\pi \pi^{*}\right)$ state $(2.30 \mathrm{eV}$, see Table 2$)$. The energy of $\mathrm{CI}_{\mathrm{twist}}$, on the other hand, is $4.43 \mathrm{eV}$, which is much higher than the energies of $\mathrm{CI}_{\mathrm{SPT}}$ and $\mathrm{CI}_{\mathrm{DPT}}$ and the $1^{1} B_{u}\left(\pi \pi^{*}\right)$ vertical excitation energy. These results indicate that $\mathrm{CI}_{\mathrm{SPT}}$ and $\mathrm{CI}_{\mathrm{DPT}}$ may be energetically accessible after vertical excitation of $\mathbf{2}$, while $\mathrm{CI}_{\text {twist }}$ is not accessible, being too high in energy.

For 1, the energies of $\mathrm{CI}_{\mathrm{SPT}}$ and $\mathrm{CI}_{\mathrm{DPT}}$ are 2.57 and $2.03 \mathrm{eV}$, respectively, and are thus close to the experimental vertical excitation energy $(2.27-2.30 \mathrm{eV}$, see Table 1). It is thus likely that these CIs are accessible after photoexcitation of $\mathbf{1}$. The CI energies of $\mathbf{1}$ are higher than the computed value of the 
$1^{1} B_{u}\left(\pi \pi^{*}\right)$ vertical excitation energy $(1.81 \mathrm{eV})$, which, however, may be the result of a significant underestimation of the latter, as discussed in Section 3.1.

The $\mathrm{CI}_{\text {twist }}$ geometry of $\mathbf{2}$ exhibits, in addition to the twist of the central $\mathrm{C}=\mathrm{C}$ double bond, a large outof-plane twisting of the $\mathrm{C}=\mathrm{C}$ bond of one of the five-membered rings, see Fig. 3c. It can be inferred from this observation that $\mathrm{CI}_{\mathrm{twist}}$ of $\mathbf{1}$ must be quite high in energy, because the out-of-plane deformation of the five-membered rings of $\mathbf{1}$ will be blocked by the fusion with the six-membered rings. $\mathrm{CI}_{\text {twist }}$ should thus be even higher in energy in $\mathbf{1}$ than in $\mathbf{2}$.

Fig. 4 shows the two singly occupied molecular orbitals at $\mathrm{CI}_{\mathrm{SPT}}$ and $\mathrm{CI}_{\mathrm{DPT}}$ of $\mathbf{1}$ as well as $\mathrm{CI}_{\text {twist }}$ of $\mathbf{2}$. The orbitals at $\mathrm{CI}_{\mathrm{SPT}}$ and $\mathrm{CI}_{\mathrm{DPT}}$ of $\mathbf{2}$ are shown in the ESI. $\dagger$ It should be noted that the orbital which is adiabatically connected with the original HOMO is now localized on only one of the ring moieties, rather than covering the central $\mathrm{C}=\mathrm{C}$ bond, as found at the $\mathrm{S}_{0}$ equilibrium geometry (cf. Fig. 2). The orbital which adiabatically emerges from the original LUMO is, on the other hand, similar to the LUMO at the $\mathrm{S}_{0}$ equilibrium geometry. Concerning $\mathrm{CI}_{\text {twist }}$ in Fig. 4, we note that the original HOMO has developed to a molecular orbital which is completely localized on the internally twisted five-membered ring, while the LUMO has developed to a molecular orbital which is delocalized over both rings.

\subsection{Potential-energy profiles of proton transfer}

For 1 and 2, the coordinate-driven minimum-energy paths for SPT have been determined by fixing one of the proton-transfer coordinates ( $s_{a}$ or $s_{b}$, see eqns (1) and (2)) and optimizing the lowest ${ }^{1} \pi \pi^{*}$ energy with respect to all other coordinates. The geometry optimizations have been performed with the CASSCF method and $C_{S}$ symmetry constraint. In $C_{s}$ symmetry, the $\mathrm{S}_{0}$ state and the ${ }^{1} \pi \pi^{*}$ state are both of ${ }^{1} A^{\prime}$ irreducible representation. The energies at the optimized geometries have been calculated with the CASPT2 method. Fig. 5 shows the resulting PE profiles of the ${ }^{1} \pi \pi^{*}$ state of $\mathbf{1}$ and $\mathbf{2}$ as a function of $s_{a}$ as solid lines with filled squares (the PE profiles along $s_{b}$ are identical due to the $C_{2 h}$ symmetry of $\mathbf{1}$ and $\mathbf{2}$ ). The PE profiles of the $\mathrm{S}_{0}$ state, calculated at the ${ }^{1} \pi \pi^{*}$-optimized geometries (dashed lines with filled 
circles), as well as at the $\mathrm{S}_{0}$-optimized geometries, determined with the MP2 method (solid lines with filled circles), are also shown. The cross indicates the proton-transfer coordinate and the energy of $\mathrm{CI}_{\mathrm{SPT}}$ discussed in Section 3.2. The $s_{a}$ value at $\mathrm{CI}_{\mathrm{SPT}}$ of $\mathbf{1}$ and $\mathbf{2}$ is 0.55 and $0.61 \AA$, respectively.

For 1, the PE profile of the ${ }^{1} \pi \pi^{*}$ state exhibits two minima with similar energies at $s_{a}=-0.4$ and $0.4 \AA$ (see Fig. 5a), corresponding to the keto form and the mono-enol form, respectively. The barrier between the keto and mono-enol minima is approximately $0.20 \mathrm{eV}$ above the keto minimum. For 2 , no barrier is found along the PE profile of the SPT in the ${ }^{1} \pi \pi^{*}$ state, see Fig. 5b. The energy profile of 2 exhibits no minimum for the keto form, but exhibits a minimum for the mono-enol form at $s_{a}=0.6 \AA$ which is significantly lower in energy than the keto form.

The energy of $\mathrm{CI}_{\mathrm{SPT}}$ is very close to the energy of the ${ }^{1} \pi \pi^{*}$ minimum of the mono-enol form on the SPT reaction path in both $\mathbf{1}$ and $\mathbf{2}$. This suggests that after the SPT in the ${ }^{1} \pi \pi^{*}$ state, the molecules can easily decay via $\mathrm{CI}_{\mathrm{SPT}}$ to the $\mathrm{S}_{0}$ state. On the minimum-energy reaction path, an energy gap of about 1.0 and $0.5 \mathrm{eV}$, respectively, remains after SPT of 1 and 2, see Fig. 5. To reach $\mathrm{CI}_{\mathrm{SPT}}$ from the minimumenergy path, an additional in-plane deformation is necessary which raises the $\mathrm{S}_{0}$ energy, leading to an intersection with the $S_{1}$ energy surface. The PE profile of the path connecting the ${ }^{1} \pi \pi^{*}$ minimum with $\mathrm{CI}_{\mathrm{SPT}}$ will qualitatively look like Fig. $6 \mathrm{a}$, where $q$ is a linear combination of internal coordinates which represents the additional in-plane motion. The character of $q$ can be inferred from the comparison of the bond lengths at the ${ }^{1} \pi \pi^{*}$ minimum of the mono-enol form and at the $\mathrm{CI}_{\mathrm{SPT}}$ structure. These two structures are represented as the circle and cross in Fig. 6a, respectively. Figs. $6 \mathrm{~b}$ and $6 \mathrm{c}$ give the specification of the relevant bond lengths of the mono-enol minimum $\left(s_{a}=0.40 \AA\right)$ and $\mathrm{CI}_{\mathrm{SPT}}\left(s_{a}=0.55\right.$ $\AA$ ) of $\mathbf{1}$. The bond lengths which exhibit major changes are indicated by the numbers which are printed in bold and blue in the figures. One can see that $\mathrm{CI}_{\mathrm{SPT}}$ is reached from the mono-enol minimum of the reaction path by the stretching of bonds in the ring which has lost a proton by the SPT. The mono-enol minimum and $\mathrm{CI}_{\mathrm{SPT}}$ of $\mathbf{2}$ exhibit similar behavior, see the ESI. $\dagger$ It is noteworthy that no out-of-plane deformation is required to reach $\mathrm{CI}_{\mathrm{SPT}}$ in the course of the proton-transfer process in $\mathbf{1}$ and $\mathbf{2}$. This 
finding is in contrast to internal conversion via ESIPT in other aromatic or heteroaromatic systems, in which a large out-of-plane torsion is required for the access to the $\mathrm{S}_{1}-\mathrm{S}_{0} \mathrm{CI} .^{56-60}$

For the proton back transfer in the $\mathrm{S}_{0}$ state (from the mono-enol form to the keto form), the PE profiles in Fig. 5 (solid lines with filled circles) predict low energy barriers of 0.30 and $0.14 \mathrm{eV}$ for 1 and 2, respectively. These barriers are easily overcome because of the substantial vibrational excess energy available after the $\mathrm{S}_{1}$ deactivation via $\mathrm{CI}_{\text {SPT }}$.

The reaction paths for excited-state DPT have been determined by CASSCF geometry optimizations in $C_{2 h}$ symmetry, using the symmetric proton-transfer coordinate $s$ as driving coordinate of the relaxed scan. This reaction path corresponds to the concerted transfer of both protons in opposite directions. DPT by two sequential SPT processes should not play a role in the photochemistry of indigo dyes, because the $\mathrm{S}_{1}$ state is efficiently deactivated via $\mathrm{CI}_{\mathrm{SPT}}$ after the SPT process from the keto form to the mono-enol form, as discussed above.

The CASPT2 PE profiles, optimized for the $1{ }^{1} B_{u}\left(\pi \pi^{*}\right)$ state as well as for the $2^{1} A_{g}\left(\pi \pi^{*}\right)$ state, are shown in Fig. 7. In contrast to SPT, the PE profiles for DPT exhibit large barriers on the reaction path from the keto form $(s<0)$ to the di-enol form $(s>0)$ for both $\mathbf{1}$ and $\mathbf{2}$. For $\mathbf{1}$, the DPT path in the $1^{1} B_{u}$ state exhibits a barrier of $1.06 \mathrm{eV}$ (energy difference between $s=-0.6 \AA$ and $s=0.0 \AA$ ). After overcoming this barrier, the molecule may switch to the $2{ }^{1} A_{g}$ state, which is below the $1{ }^{1} B_{u}$ state for $s>$ 0 . The energy gap between the $2^{1} A_{g}$ state and the $1{ }^{1} A_{g}\left(\mathrm{~S}_{0}\right)$ state becomes quite small in the di-enol form $(s>0)$, which indicates the possibility of nonradiative decay through $\mathrm{CI}_{\mathrm{DPT}}$. However, this decay mechanism is unlikely to play a role in the photochemistry of $\mathbf{1}$, because of the large barrier separating the keto and di-enol forms. For 2, the PE barrier for DPT can be estimated from the difference between the $1^{1} B_{u}$ energy at $s=-0.6 \AA$ and the $2^{1} A_{g}$ energy at $s=0.0 \AA$, yielding $0.75 \mathrm{eV}$. The DPT of 2 is thus also inhibited by a significant barrier.

The barrier for the photoinduced SPT reaction in indigo is thus definitely much lower than the barrier for DPT. This finding can be explained by considering the change of the molecular structure along the reaction paths for the two types of ESIPT. In the geometry optimizations of the reaction paths, it is 
found that the distance between the adjacent $\mathrm{N}$ and $\mathrm{O}$ atoms becomes much shorter at intermediate PT structures than at the reactant and product structures. In other words, the adjacent $\mathrm{N}-\mathrm{H}$ and $\mathrm{C}=\mathrm{O}$ groups have to approach each other during the ESIPT process. For SPT, this is achieved by a rocking of the proton donor and acceptor moieties with respect to the central $\mathrm{C}=\mathrm{C}$ bond. The rocking motion allows the adjacent $\mathrm{N}-\mathrm{H}$ and $\mathrm{C}=\mathrm{O}$ groups to approach each other without too much strain. For DPT, on the other hand, the adjacent $\mathrm{N}-\mathrm{H}$ and $\mathrm{C}=\mathrm{O}$ groups on both sides of the molecule have to approach each other simultaneously. Since the rocking of the two rings is excluded in the case of DPT, the N-H and C=O groups have to reduce their distance by other motions, such as shortening of the central $\mathrm{C}=\mathrm{C}$ bond or deformation of the $\mathrm{C}=\mathrm{O}$ group. These deformations raise the energy of the intermediate DPT structure considerably, resulting in the high barrier for DPT.

The high barrier for DPT predicted by the CASPT2 calculations is confirmed by CC2 calculations of the PE profiles. Fig. 8 shows the PE surface of the lowest ${ }^{1} \pi \pi^{*}\left(2^{1} A^{\prime}\right)$ state of 1 in the Franck-Condon (FC) region as a function of $s_{a}$ and $s_{b}$. The other coordinates have been optimized for the ${ }^{1} \pi \pi^{*}$ state with $C_{s}$ symmetry constraint at the CC2 level. The SPT paths in Fig. 8 were optimized by fixing either $s_{a}$ or $s_{b}$ (solid line with filled squares and dashed line, respectively), whereas the DPT path was optimized with the constraint $s_{a}=s_{b}=s$ (solid line with open squares), resulting in the $1{ }^{1} B_{u}$ state of $C_{2 h}$ symmetry. According to these CC2 PE profiles, the barrier of the SPT path is $0.08 \mathrm{eV}$, which is much lower than the barrier of the DPT path, which is estimated as $0.78 \mathrm{eV}$. Furthermore, the DPT reaction path is unstable near the barrier top, i.e., the DPT intermediate structure is a maximum rather than a saddle point in the two-dimensional $\left(s_{a}, s_{b}\right)$ space.

As Fig. 8 shows, the CC2 PE profiles computed in the $C_{2 h}$ and $C_{s}$ symmetries, respectively, cross at one point on the surface $\left(s_{a}=s_{b}=-0.58 \AA\right.$ ), that is, the minima of the $C_{2 h}$ and $C_{s} \mathrm{PE}$ curves correspond to the same geometry. The geometries of the $C_{2 h}$ and $C_{s}$ minima of the CASSCF-optimized PE curves in the FC region (Figs. 5 and 7), on the other hand, correspond to different geometries. This discrepancy presumably is the consequence of the lack of dynamical electron correlation in the CASSCF geometry optimization. 
The computational results thus indicate that the ${ }^{1} \pi \pi^{*}$ excited state of indigo can efficiently deactivate through $\mathrm{CI}_{\mathrm{SPT}}$, which explains the short excited-state lifetime of indigo dyes. The calculations clearly show that excited-state SPT takes place, rather than DPT. This result is consistent with the interpretation of time-resolved pump-probe spectra by Iwakura et al., which exhibit the transient formation of the mono-enol structure and ultrafast return to the keto form without the formation of the di-enol structure. ${ }^{36,37}$

The dependence of the excited-state lifetime of indigo derivatives on solvent polarity ${ }^{29}$ can also be explained by the SPT mechanism. The initial keto structure of the photoreaction has no dipole moment because of the $C_{2 h}$ symmetry, while the mono-enol structure formed by SPT has a non-zero dipole moment, since the $C_{2 h}$ symmetry is broken. It is therefore expected that the electrostatic interaction between the indigo dye and a polar solvent stabilizes the mono-enol form (the product of SPT) more than the keto form (the reactant of SPT). This solvent effect lowers the energy barrier for SPT and thus shortens the excited-state lifetime. A similar stabilization of the ESIPT product by solute-solvent electrostatic interaction has been computationally demonstrated for an aminomethylnaphthol compound in acetonitrile. ${ }^{73}$

The biexponential decay of the fluorescence of indigo ${ }^{24,30}$ may be attributed to the existence of two $\mathrm{S}_{1}$ minima, corresponding to the keto and mono-enol forms, respectively (see Fig. 5a). In particular, the biexponential decay has been observed even for indigo derivatives possessing only one intramolecular hydrogen bond, ${ }^{30}$ which again supports the formation of the mono-enol structure in the $S_{1}$ state. The present CASPT2 calculations predict fluorescence emission energies of $\mathbf{1}$ (1.56 and $0.98 \mathrm{eV}$ at the keto and mono-enol minima, respectively) which are significantly lower than the experimental value estimated from the fluorescence wavelength in solution $(1.90 \mathrm{eV}, 653 \mathrm{~nm}){ }^{24,30}$ This discrepancy is most likely the consequence of a significant underestimation of the ${ }^{1} \pi \pi^{*}$ excitation energy at the CASPT2 level due to an insufficiently large active space in the underlying CASSCF calculation and possibly also due to the lack of dynamical electron correlation in the geometry optimizations. 


\subsection{Potential-energy profiles of trans $\rightarrow$ cis photoisomerization}

The reaction path for the trans $\rightarrow$ cis photoisomerization of $\mathbf{2}$ has been determined by fixing the twisting coordinate $\varphi$ (eqn (3)) and optimizing the energy of the excited state with respect to the other coordinates with the CASSCF method. We have optimized the reaction path in the ${ }^{1} \pi \pi^{*}$ state as well as in the ${ }^{1} \mathrm{n} \pi^{*}$ state which corresponds to the excitation from the lone pair of one $\mathrm{O}$ atom. Single-point CASPT2 energies have been calculated at the optimized geometries. The resulting PE profiles are shown in Fig. 9. Solid lines with filled squares and open triangles give the optimized PE profiles in the ${ }^{1} \pi \pi^{*}$ state and in the ${ }^{1} \mathrm{n} \pi^{*}$ state, respectively. The dashed line with open triangles represents the ${ }^{1} \mathrm{n} \pi^{*}$ energies at the ${ }^{1} \pi \pi^{*}$-optimized geometries. The dashed line with filled circles gives the $\mathrm{S}_{0}$ energies at the ${ }^{1} \mathrm{n} \pi^{*}-$ optimized geometries. The cross indicates the twist angle and the energy of the $\mathrm{CI}_{\mathrm{twist}}$ structure (see Fig. $3 c)$.

As shown in Fig. 9, the ${ }^{1} \pi \pi^{*}$ PE curve exhibits two minima and a substantial energy barrier separating the trans isomer (around $\varphi=0^{\circ}$ ) and the cis isomer (around $\varphi=135^{\circ}$ ). The energy of the cis isomer is higher than the minimum of the trans isomer by more than $1 \mathrm{eV}$. The barrier height, estimated from the energy difference of the ${ }^{1} \pi \pi^{*}$ state at $\varphi=0^{\circ}$ and $\varphi=90^{\circ}$, is $1.36 \mathrm{eV}$. The energy of $\mathrm{CI}_{\mathrm{twist}}$, with a twist angle of $110^{\circ}$, is even higher than the energy of the barrier. These results indicate that the trans $\rightarrow$ cis photoisomerization is most likely not involved in the internal-conversion dynamics of indigo.

The ${ }^{1} \mathrm{n} \pi^{*}$ state may be populated during the photoisomerization of 2 through the crossing with the ${ }^{1} \pi \pi^{*}$ state near $\varphi=30^{\circ}$, see Fig. 9. However, the PE curve of the ${ }^{1} \mathrm{n} \pi^{*}$ state also exhibits a barrier. The barrier height can be estimated from the difference between the ${ }^{1} \pi \pi^{*}$ energy at $\varphi=0^{\circ}$ and the ${ }^{1} \mathrm{n} \pi^{*}$ energy at $\varphi=$ $120^{\circ}$, resulting in $0.64 \mathrm{eV}$. For $\mathbf{1}$, photoisomerization in the ${ }^{1} \mathrm{n} \pi^{*}$ state is more difficult than for 2. According to the vertical excitation energies of Tables 1 and 2 , the ${ }^{1} n \pi^{*}$ state in $\mathbf{1}$ is higher in energy than in $\mathbf{2}$, while the ${ }^{1} \pi \pi^{*}$ state is more stable in $\mathbf{1}$ than in $\mathbf{2}$. If the shape of the twisting energy profiles in $\mathbf{1}$ and $\mathbf{2}$ is similar, the energy barrier of the ${ }^{1} \mathrm{n} \pi^{*}$ state in $\mathbf{1}$ is higher than the barrier in $\mathbf{2}$. 
The PE profiles of indigo illustrated in Fig. 9 are quite different from those of other molecules undergoing photoisomerization via the twisting of a double bond, which exhibit effective reaction paths with low barriers leading to CIs. In addition, the change of other geometry parameters during the twisting is very different. Ethene is the best known example of photoisomerization via double-bond twisting. ${ }^{63,74-78}$ After photoexcitation of ethene to the ${ }^{1} \pi \pi^{*}$ state, twisting, pyramidization at one of the C atoms, as well as migration of $\mathrm{H}$ atoms occur and lead to a low-lying $\mathrm{CI}$ of the $\mathrm{S}_{1}$ state with the ground state. Photoexcited indigo, on the other hand, exhibits very little pyramidization at the central $\mathrm{C}$ atoms as well as very little out-of-plane deformation of the rings along the twisting paths. Rather, out-of-plane deformation of one of the five-membered rings is required to reach the $\mathrm{CI}_{\mathrm{twist}}$ geometry (see Fig. $3 \mathrm{c}$ ), which considerably increases the ${ }^{1} \pi \pi^{*}$ energy (see Fig. 9). The difference between ethene and indigo is thus the rigidity of the five-membered rings, which prevents pyramidization at the central $\mathrm{C}$ atoms of indigo. To support this conclusion, we have calculated PE profiles for twisting in the lowest ${ }^{1} \pi \pi^{*}$ state of the compounds shown in Scheme 2. In compound 3, the $\mathrm{C}$ atoms of the $\mathrm{C}=\mathrm{C}$ double bonds of the fivemembered rings of $\mathbf{2}$ are saturated with $\mathrm{H}$ atoms, which eliminates the double bonds. Compound $\mathbf{4}$ is the so-called H-chromophore of indigo, in which the rings are replaced by flexible substituents. ${ }^{38,39}$ Relaxed scans for twisting were carried out for 3 and $\mathbf{4}$ with the CASSCF method, using the $(6,6 / 6 \pi)$ active space and state averaging of lowest two singlet states $\left(\mathrm{S}_{0}\right.$ and $\left.{ }^{1} \pi \pi^{*}\right)$, and then CASPT2 energies were calculated at the optimized geometries. Two $\mathrm{p}$ orbitals on the $\mathrm{N}$ atoms had to be excluded from the active space, because they strongly mix with the $\sigma$ orbital of the central $\mathrm{C}=\mathrm{C}$ bond. Fig. 10 shows the resulting PE profiles of 3 and $\mathbf{4}$. The PE profile of the ${ }^{1} \pi \pi^{*}$ state of $\mathbf{2}$, calculated with the $(10,10 / 10 \pi)$ active space, in which the two $\mathrm{p}$ orbitals on the $\mathrm{N}$ atoms also were excluded, is shown in Fig. 10a for comparison. In contrast to $\mathbf{2}$, the energy profiles of the reaction paths of $\mathbf{3}$ and $\mathbf{4}$ exhibit a very small energy gap between the ${ }^{1} \pi \pi^{*}$ state and the $\mathrm{S}_{0}$ state at twisted structures (see Figs. $10 \mathrm{~b}$ and $10 \mathrm{c}$ ), which indicates that trans $\rightarrow$ cis photoisomerization and internal conversion via a ${ }^{1} \pi \pi^{*}-\mathrm{S}_{0}$ CI readily occur in 3 and 4. The PE profile of 3 exhibits a low barrier between the FC region $\left(\varphi=0^{\circ}\right)$ and the twisted 
structure $\left(\varphi=90^{\circ}\right)$, while the PE profile of 4 is almost barrierless. (The CASSCF optimizations of 4 could not be converged for $\varphi>75^{\circ}$, because a ${ }^{1} \pi \pi^{*}-\mathrm{S}_{0}$ CI was encountered.) These findings support the conjecture that the rigidity of the five-membered rings of $\mathbf{2}$, enhanced by the $\mathrm{C}=\mathrm{C}$ double bonds, is of significance for the suppression of the trans $\rightarrow$ cis photoisomerization. For $\mathbf{1}$, the six-membered rings fused with the five-membered rings are expected to enhance the rigidity even further.

The fact that the trans $\rightarrow$ cis photoisomerization of indigo is largely suppressed explains the lack of detection of the cis isomer of indigo in the early experiments. ${ }^{5,6}$ The absence of the $\mathrm{C}=\mathrm{C}$ twisting is also consistent with the experimental observation that the excited-state lifetimes of indigo derivatives in solution are independent of the solvent viscosity. ${ }^{26,29}$

The present work has been focused on the singlet states of indigo, motivated by the very low quantum yield of intersystem crossing in indigo. ${ }^{23-25}$ For some indigo derivatives such as thioindigo, however, it has been proposed that triplet states are involved as intermediates in the trans $\rightarrow$ cis photoisomerization. ${ }^{15-22}$ The first-principles investigation of the photochemistry in the triplet manifold should therefore be of interest for these derivatives of indigo.

\section{Conclusions}

The present work is the first systematic computational study of the molecular mechanisms of the photostability of indigo with $a b$ initio methods. Fig. 11 shows the overview of the photochemistry of indigo which has been elucidated in the present work. The CASSCF, CASPT2 and CC2 calculations performed in the present work have revealed that the mechanism of the exceptional photostability of indigo is the ESIPT reaction along the hydrogen bonds between the adjacent $\mathrm{C}=\mathrm{O}$ and $\mathrm{N}-\mathrm{H}$ groups. The trans $\rightarrow$ cis photoisomerization via the twisting of the central $\mathrm{C}=\mathrm{C}$ bond is strongly suppressed by the rigidity of the five-membered rings. The ESIPT process has been shown to be a SPT reaction, rather than a concerted DPT reaction. A CI which is accessible via the SPT process causes very fast nonradiative decay from the $S_{1}$ state to the $S_{0}$ state. The mechanistic picture emerging from the present 
computational results explains the experimental observations on the photophysics of indigo, such as the missing of the cis isomer, ${ }^{5,6}$ the very short excited-state lifetime, ${ }^{23,24,26-30}$ its dependence on solvent

polarity, ${ }^{29}$ its independence of solvent viscosity, ${ }^{26,29}$ as well as the transient formation of the mono-enol form in the excited state. ${ }^{36,37}$

We would like to emphasize that the presence of ESIPT and the absence of trans $\rightarrow$ cis photoisomerization should be considered separately and that both of them are essential for the photostability of indigo. It has been argued that the trans $\rightarrow$ cis photoisomerization of indigo is prevented by ultrafast deactivation of the photoexcited state via ESIPT. ${ }^{26,34,35}$ However, the photoisomerization would compete with the ESIPT process if it were energetically possible, because the former as well as the latter can take place very efficiently via CIs. If the ultrafast deactivation via ESIPT would not exist in indigo, on the other hand, the excited-state lifetime would be much longer, which would enhance the quantum yield of destructive photoreactions.

The investigation of the photophysics of indigo derivatives and the analysis of the similarities with and the differences from the photophysics of indigo would lead to further understanding of the mechanism of the photostability. This is an interesting topic of future research.

\section{Acknowledgements}

This work has been supported by the Deutsche Forschungsgemeinschaft (DFG) through SFB 749. S.Y. also acknowledges support by a fellowship of the Alexander von Humboldt Foundation. A.L.S. acknowledges support through a visitor grant of the DFG Cluster of Excellence "Munich-Centre for Advanced Photonics" (www.munich-photonics.de). We thank the Leibniz-Rechenzentrum der Bayerischen Akademie der Wissenschaften for substantial allocation of computing time. 


\section{Captions of schemes/figures}

Scheme 1 Molecular structures of 1 (indigo) and 2 (bispyrroleindigo).

Scheme 2 Molecular structures of 3 and 4.

Fig. 1 Photoinduced reactions studied in this paper. (a) ESIPT of 1, (b) ESIPT of 2 and (c) trans $\rightarrow$ cis photoisomerization of $\mathbf{2}$. See eqns (1)-(3) for the definition of the proton-transfer coordinates $s_{a}$ and $s_{b}$ as well as the twisting coordinate $\varphi$.

Fig. 2 HOMO (left) and LUMO (right) at the $\mathrm{S}_{0}$ equilibrium geometry of (a) 1 and (b) $\mathbf{2}$, calculated at the RHF level.

Fig. 3 CASSCF-optimized geometries of ${ }^{1} \pi \pi^{*}-\mathrm{S}_{0}$ CIs. (a) $\mathrm{CI}_{\mathrm{SPT}}$ of $\mathbf{1}$ and $\mathbf{2}$, (b) $\mathrm{CI}_{\mathrm{DPT}}$ of 1 and $\mathbf{2}$ and (c) $\mathrm{CI}_{\text {twist }}$ of 2. The numbers give the CASPT2 energies of the CIs relative to the energy of the $\mathrm{S}_{0}$ minimum. The $(10,10 / 10 \pi)$ and $(14,12 / 12 \pi)$ active spaces were used in the calculations for $\mathbf{1}$ and $\mathbf{2}$, respectively.

Fig. 4 Singly occupied molecular orbitals at the ${ }^{1} \pi \pi^{*}-\mathrm{S}_{0}$ CI, calculated at the CASSCF level. (a) CI SPT $_{\text {of }}$ 1, (b) $\mathrm{CI}_{\mathrm{DPT}}$ of 1 and (c) $\mathrm{CI}_{\mathrm{twist}}$ of 2. 
Fig. 5 Potential-energy profiles (relaxed scans) of the SPT reaction in the ${ }^{1} \pi \pi^{*}$ state as well as in the $\mathrm{S}_{0}$ state as a function of the proton-transfer coordinate $s_{a}$, for (a) 1 and (b) $\mathbf{2}$, calculated at the CASPT2 level. Full line with filled squares (filled circles): ${ }^{1} \pi \pi^{*}\left(\mathrm{~S}_{0}\right)$ energies at CASSCF-optimized (MP2optimized) geometries. Dashed line with filled circles: $\mathrm{S}_{0}$ energies at ${ }^{1} \pi \pi^{*}$-optimized geometries. The cross indicates the CASPT2 energy of $\mathrm{CI}_{\mathrm{SPT}}$.

Fig. 6 (a) Schematic picture of the potential-energy profile of the SPT reaction connecting the ${ }^{1} \pi \pi^{*}$ minimum with $\mathrm{CI}_{\mathrm{SPT}}$. Left part shows the coordinate-driven minimum-energy path along the protontransfer coordinate $s_{a}$, while right part shows a cut of the potential-energy surface along another internal coordinate $q$, which represents additional in-plane motion, at the $s_{a}$ value of $\mathrm{CI}_{\mathrm{SPT}}$. Circle and cross indicate the ${ }^{1} \pi \pi^{*}$ minimum of mono-enol form and $\mathrm{CI}_{\mathrm{SPT}}$, respectively. (b, c) Bond lengths (in $\AA$ ) of $\mathbf{1}$ at (b) the mono-enol minimum of the SPT path in the ${ }^{1} \pi \pi^{*}$ state $\left(s_{a}=0.40 \AA\right)$ and (c) at $\mathrm{CI}_{\mathrm{SPT}}\left(s_{a}=0.55 \AA\right)$. Bold blue letters indicate bond lengths which differ by more than $0.030 \AA$ between panels $\mathrm{b}$ and $\mathrm{c}$.

Fig. 7 Potential-energy profiles (relaxed scans) of the DPT reaction in the ${ }^{1} \pi \pi^{*}\left(1^{1} B_{u}\right.$ and $\left.2^{1} A_{g}\right)$ states as a function of the proton-transfer coordinate $s\left(=s_{a}=s_{b}\right)$, calculated at the CASPT2 level, for (a) 1 and (b) 2. Full line with open squares (gray squares): $1{ }^{1} B_{u}\left(2^{1} A_{g}\right)$ energies at CASSCF-optimized geometries. Dashed line with open circles (gray circles): $\mathrm{S}_{0}$ energies at $1{ }^{1} B_{u}$-optimized $\left(2^{1} A_{g}\right.$-optimized) geometries.

Fig. 8 Potential-energy surface of the ESIPT process in the lowest ${ }^{1} \pi \pi^{*}$ state of $\mathbf{1}$ as a function of the proton-transfer coordinates $s_{a}$ and $s_{b}$ in the Franck-Condon region, calculated at the CC2 level. Full line with open squares: DPT path, optimized with the constraint $s_{a}=s_{b}$. Full line with filled squares: SPT path, optimized for fixed $s_{a}\left(s_{b}\right.$ is optimized). Dashed: SPT path, optimized for fixed $s_{b}\left(s_{a}\right.$ is optimized). 
Fig. 9 Potential-energy profiles of trans $\rightarrow$ cis photoisomerization of 2 in the ${ }^{1} \pi \pi^{*}$ state as well as in the ${ }^{1} \mathrm{n} \pi^{*}$ state as a function of the twisting coordinate $\varphi$, calculated at the CASPT2 level. Full line with filled squares (open triangles): ${ }^{1} \pi \pi^{*}\left({ }^{1} \mathrm{n} \pi^{*}\right)$ energies at CASSCF-optimized geometries. Dashed line with open triangles: ${ }^{1} \mathrm{n} \pi^{*}$ energies at ${ }^{1} \pi \pi^{*}$-optimized geometries. Dashed line with filled circles: $\mathrm{S}_{0}$ energies at ${ }^{1} \mathrm{n} \pi^{*}-$ optimized geometries. The cross indicates the CASPT2 energy of $\mathrm{CI}_{\text {twist }}$.

Fig. 10 Potential-energy profiles of trans $\rightarrow$ cis photoisomerization in the ${ }^{1} \pi \pi^{*}$ state as function of the twisting coordinate $\varphi$, for (a) 2, (b) 3 and (c) 4, calculated at the CASPT2 level. Full lines with filled squares: ${ }^{1} \pi{ }^{*}$ energies at CASSCF-optimized geometries. Dashed lines with filled circles: $\mathrm{S}_{0}$ energies at ${ }^{1} \pi \pi^{*}$-optimized geometries.

Fig. 11 Overview of the mechanism of the photostability of indigo. 
Table 1 Vertical excitation energies (in $\mathrm{eV}$ ) and oscillator strengths (in parentheses) of the low-lying singlet excited states of $\mathbf{1}$

\begin{tabular}{llll}
\hline State & CC2 & CASPT2 $(14,14 / 14 \pi)$ & Exp. $^{a}$ \\
\hline $1{ }^{1} B_{u}\left(\pi \pi^{*}\right)$ & $2.34(0.375)$ & $1.81(0.183)$ & $2.27-2.30$ \\
$1^{1} B_{g}\left(\mathrm{n} \pi^{*}\right)$ & $3.06(0)$ & & \\
$2{ }^{1} A_{g}\left(\pi \pi^{*}\right)$ & $3.22(0)$ & & \\
$1^{1} A_{u}\left(\mathrm{n} \pi^{*}\right)$ & $3.52\left(3.8 \times 10^{-6}\right)$ & & \\
${ }^{1} A_{g}\left[\left(\pi^{*}\right)^{2}\right]^{b}$ & 2.38 & & \\
\hline
\end{tabular}

${ }^{a}$ Absorption spectrum in the gas phase. ${ }^{40,70-72}$

${ }^{b}$ Calculated using the RHF wave function for the doubly-excited configuration as the reference of the CC2 calculation. 
Table 2 Vertical excitation energies (in $\mathrm{eV}$ ) and oscillator strengths (in parentheses) of the low-lying singlet excited states of $\mathbf{2}$

\begin{tabular}{|c|c|c|c|c|}
\hline State & $\mathrm{CC} 2$ & CASPT2 $(14,12 / 12 \pi)$ & CASPT2 $(14,12 / 10 \pi 2 n)$ & Exp. $^{a}$ \\
\hline $1{ }^{1} B_{u}\left(\pi \pi^{*}\right)$ & $2.68(0.268)$ & $2.30(0.208)$ & $2.45(0.190)$ & 2.57 \\
\hline $1{ }^{1} B_{g}\left(\mathrm{n} \pi^{*}\right)$ & $2.85(0)$ & & $2.30(0)$ & \\
\hline $1^{1} A_{u}\left(\mathrm{n} \pi^{*}\right)$ & $3.32\left(8.6 \times 10^{-6}\right)$ & & $2.53\left(1.8 \times 10^{-5}\right)$ & \\
\hline $2^{1} A_{g}\left(\pi \pi^{*}\right)$ & $3.35(0)$ & & $2.97(0)$ & \\
\hline${ }^{1} A_{g}\left[\left(\pi^{*}\right)^{2}\right]^{b}$ & 2.45 & & & \\
\hline
\end{tabular}

${ }^{a}$ Absorption spectrum of tetramethylated $\mathbf{2}$ in the gas phase. ${ }^{40}$

${ }^{b}$ See footnote b of Table 1 . 


\section{References}

[1] J. Balfour-Paul, Indigo, British Museum Press, London, 1998.

[2] E. S. B. Ferreira, A. N. Hulme, H. McNab and A. Quye, Chem. Soc. Rev., 2004, 33, 329-336.

[3] S. Fantacci, A. Amat and A. Sgamellotti, Acc. Chem. Res., 2010, 43, 802-813.

[4] M. J. Melo and A. Claro, Acc. Chem. Res., 2010, 43, 857-866.

[5] W. R. Brode, E. G. Pearson and G. M. Wyman, J. Am. Chem. Soc., 1954, 76, 1034-1036.

[6] G. M. Wyman, Chem. Rev., 1955, 55, 625-657.

[7] G. M. Wyman and W. R. Brode, J. Am. Chem. Soc., 1951, 73, 1487-1493.

[8] J. Weinstein and G. M. Wyman, J. Am. Chem. Soc., 1956, 78, 4007-4010.

[9] R. Pummerer and G. Marondel, Chem. Ber., 1960, 93, 2834-2839.

[10] G. M. Wyman and A. F. Zenhäusern, J. Org. Chem., 1965, 30, 2348-2352.

[11] H. Güsten, J. Chem. Soc. D, 1969, 133-134.

[12] D. L. Ross, J. Blanc and F. J. Matticoli, J. Am. Chem. Soc., 1970, 92, 5750-5752.

[13] G. M. Wyman and B. M. Zarnegar, J. Phys. Chem., 1973, 77, 831-837.

[14] Y. Omote, S. Imada, R. Matsuzaki, K. Fujiki, T. Nishio and C. Kashima, Bull. Chem. Soc. Jpn., 1979, 52, 3397-3399.

[15] C. R. Giuliano, L. D. Hess and J. D. Margerum, J. Am. Chem. Soc., 1968, 90, 587-594.

[16] G. M. Wyman, B. M. Zarnegar and D. G. Whitten, J. Phys. Chem., 1973, 77, 2584-2586. 
[17] A. D. Kirsch and G. M. Wyman, J. Phys. Chem., 1975, 79, 543-544.

[18] D. Schulte-Frohlinde, H. Herrmann and G. M. Wyman, Z. Phys. Chem., 1976, 101, 115-121.

[19] A. D. Kirsch and G. M. Wyman, J. Phys. Chem., 1977, 81, 413-420.

[20] K. H. Grellmann and P. Hentzschel, Chem. Phys. Lett., 1978, 53, 545-551.

[21] H. Görner and D. Schulte-Frohlinde, Chem. Phys. Lett., 1979, 66, 363-369.

[22] Y. Maeda, T. Okada, N. Mataga and M. Irie, J. Phys. Chem., 1984, 88, 1117-1119.

[23] J. Seixas de Melo, A. P. Moura and M. J. Melo, J. Phys. Chem. A, 2004, 108, 6975-6981.

[24] J. S. Seixas de Melo, R. Rondão, H. D. Burrows, M. J. Melo, S. Navaratnam, R. Edge and G. Voss, ChemPhysChem, 2006, 7, 2303-2311.

[25] J. S. Seixas de Melo, H. D. Burrows, C. Serpa and L. G. Arnaut, Angew. Chem., Int. Ed., 2007, 46, 2094-2096.

[26] T. Kobayashi and P. M. Rentzepis, J. Chem. Phys., 1979, 70, 886-892.

[27] E. Lill, P. Hefferle and F. Dörr, Appl. Phys., 1980, 22, 197-200.

[28] T. Elsaesser, W. Kaiser and W. Lüttke, J. Phys. Chem., 1986, 90, 2901-2905.

[29] Y. Nagasawa, R. Taguri, H. Matsuda, M. Murakami, M. Ohama, T. Okada and H. Miyasaka, Phys. Chem. Chem. Phys., 2004, 6, 5370-5378.

[30] J. Seixas de Melo, R. Rondão, H. D. Burrows, M. J. Melo, S. Navaratnam, R. Edge and G. Voss, J. Phys. Chem. A, 2006, 110, 13653-13661.

[31] R. Rondão, J. S. Seixas de Melo and G. Voss, ChemPhysChem, 2010, 11, 1903-1908. 
[32] R. Rondão, J. S. Seixas de Melo, V. D. B. Bonifácio and M. J. Melo, J. Phys. Chem. A, 2010, 114, $1699-1708$.

[33] S. A. Krysanov and M. V. Alfimov, J. Photochem., 1983, 23, 329-333.

[34] G. M. Wyman, J. Chem. Soc. D, 1971, 1332-1334.

[35] G. M. Wyman and B. M. Zarnegar, J. Phys. Chem., 1973, 77, 1204-1207.

[36] I. Iwakura, A. Yabushita and T. Kobayashi, Chem. Lett., 2009, 38, 1020-1021.

[37] I. Iwakura, A. Yabushita and T. Kobayashi, Chem. Phys. Lett., 2010, 484, 354-357.

[38] M. Klessinger and W. Lüttke, Tetrahedron, 1963, 19, Suppl.2, 315-335.

[39] S. Dähne and D. Leupold, Angew. Chem., Int. Ed., 1966, 5, 984-993.

[40] E. Wille and W. Lüttke, Angew. Chem., Int. Ed., 1971, 10, 803-804.

[41] E. Wille and W. Lüttke, Chem. Ber., 1973, 106, 3240-3257.

[42] J. Fabian and G. Tröger-Naake, Int. J. Quantum Chem., 1977, 11, 259-269.

[43] M. Klessinger, Dyes Pigm., 1982, 3, 235-241.

[44] P. C. Chen, Int. J. Quantum Chem., 1996, 60, 681-687.

[45] L. Serrano-Andrés and B. O. Roos, Chem.-Eur. J., 1997, 3, 717-725.

[46] D. Guillaumont and S. Nakamura, Dyes Pigm., 2000, 46, 85-92.

[47] Y. S. Xue, X. D. Gong, H. M. Xiao and H. Tian, Acta Chim. Sinica, 2004, 62, 963-968.

[48] D. Jacquemin, J. Preat, V. Wathelet and E. A. Perpète, J. Chem. Phys., 2006, 124, 074104.

[49] E. A. Perpète and D. Jacquemin, J. Mol. Struct. (THEOCHEM), 2009, 914, 100-105. 
[50] J. Sühnel and K. Gustav, Mol. Photochem., 1977, 8, 437-458.

[51] J. Abe, Y. Nagasawa and H. Takahashi, J. Chem. Phys., 1989, 91, 3431-3434.

[52] G. Grimme, S. Grimme, P. G. Jones and P. Boldt, Chem. Ber., 1993, 126, 1015-1021.

[53] P. Celani and H.-J. Werner, J. Chem. Phys., 2000, 112, 5546-5557.

[54] O. Christiansen, H. Koch and P. Jørgensen, Chem. Phys. Lett., 1995, 243, 409-418.

[55] Conical Intersections: Electronic Structure, Dynamics and Spectroscopy, ed. W. Domcke, D. R. Yarkony and H. Köppel, World Scientific, Singapore, 2004.

[56] A. L. Sobolewski, W. Domcke and C. Hättig, J. Phys. Chem. A, 2006, 110, 6301-6306.

[57] A. Migani, M. J. Bearpark, M. Olivucci and M. A. Robb, J. Am. Chem. Soc., 2007, 129, 37033713.

[58] J. D. Coe, B. G. Levine and T. J. Martínez, J. Phys. Chem. A, 2007, 111, 11302-11310.

[59] A. L. Sobolewski and W. Domcke, J. Phys. Chem. A, 2007, 111, 11725-11735.

[60] M. Barbatti, A. J. A. Aquino, H. Lischka, C. Schriever, S. Lochbrunner and E. Riedle, Phys. Chem. Chem. Phys., 2009, 11, 1406-1415.

[61] N. L. Doltsinis and D. Marx, Phys. Rev. Lett., 2002, 88, 166402.

[62] P. A. Hunt and M. A. Robb, J. Am. Chem. Soc., 2005, 127, 5720-5726.

[63] B. G. Levine and T. J. Martínez, Annu. Rev. Phys. Chem., 2007, 58, 613-634.

[64] B. O. Roos and K. Andersson, Chem. Phys. Lett., 1995, 245, 215-223.

[65] F. Weigend and M. Häser, Theor. Chem. Acc., 1997, 97, 331-340.

[66] C. Hättig and F. Weigend, J. Chem. Phys., 2000, 113, 5154-5161. 
[67] T. H. Dunning, Jr., J. Chem. Phys., 1989, 90, 1007-1023.

[68] H.-J. Werner et al., MOLPRO, version 2006.1, a package of ab initio programs, 2006, see http://www.molpro.net.

[69] R. Ahlrichs, M. Bär, M. Häser, H. Horn and C. Kölmel, Chem. Phys. Lett., 1989, 162, 165-169.

[70] S. E. Sheppard and P. T. Newsome, J. Am. Chem. Soc., 1942, 64, 2937-2946.

[71] P. W. Sadler, J. Org. Chem., 1956, 21, 316-318.

[72] M. Klessinger and W. Lüttke, Chem. Ber., 1966, 99, 2136-2145.

[73] S. Yamazaki and S. Kato, Chem. Phys. Lett., 2004, 386, 414-418.

[74] V. Bonačić-Koutecký, P. Bruckmann, P. Hiberty, J. Koutecký, C. Leforestier and L. Salem, Angew. Chem., Int. Ed., 1975, 14, 575-576.

[75] E. M. Evleth and A. Sevin, J. Am. Chem. Soc., 1981, 103, 7414-7422.

[76] I. Ohmine, J. Chem. Phys., 1985, 83, 2348-2362.

[77] R. P. Krawczyk, A. Viel, U. Manthe and W. Domcke, J. Chem. Phys., 2003, 119, 1397-1411.

[78] M. Barbatti, M. Ruckenbauer and H. Lischka, J. Chem. Phys., 2005, 122, 174307. 
Scheme 1

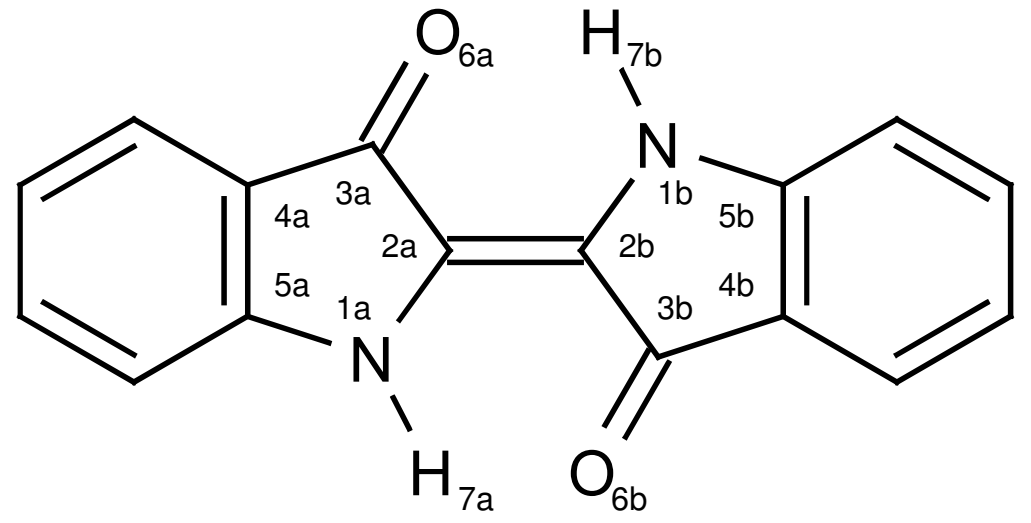

1

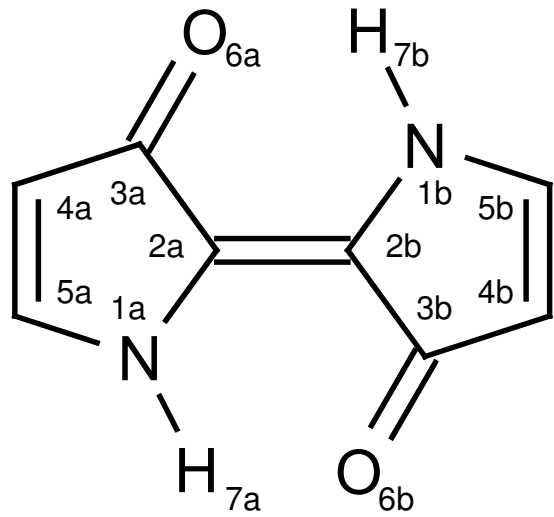

2 
Scheme 2

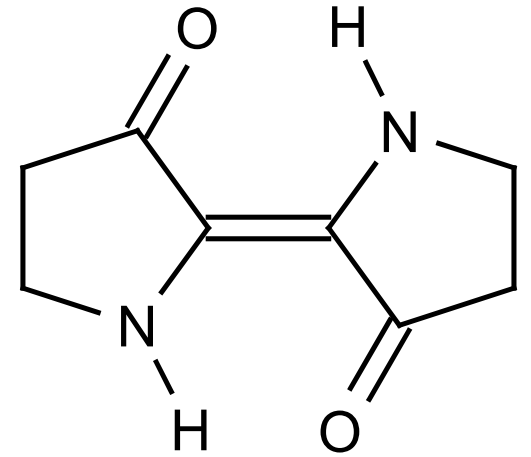

3

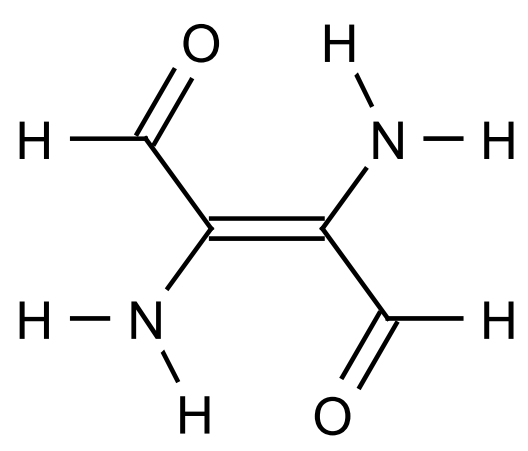

4 
Fig. 1

(a) ESIPT of 1
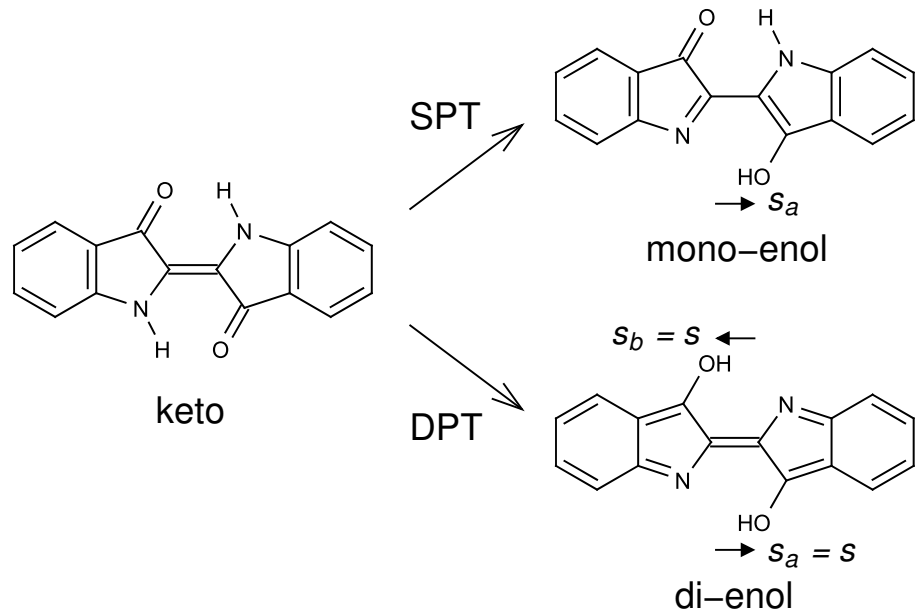

(b) ESIPT of 2
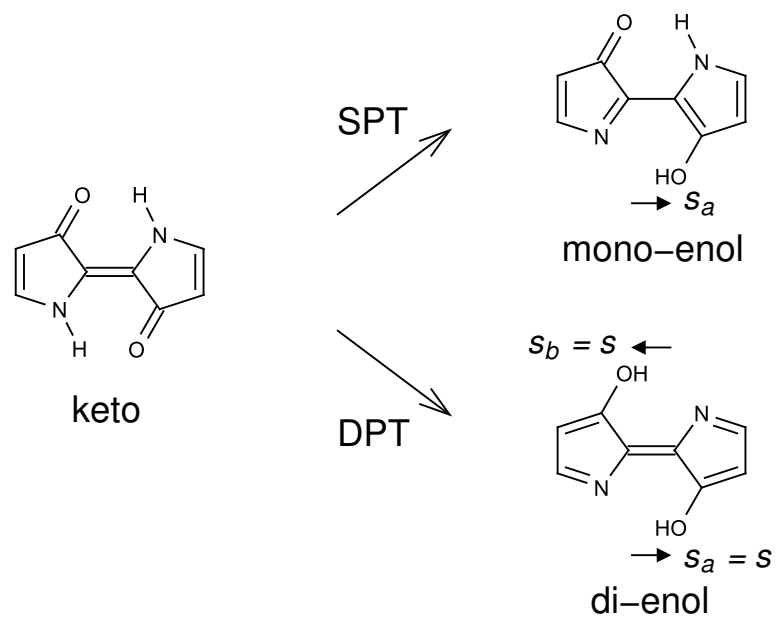

(c) trans-cis photoisomerization of 2
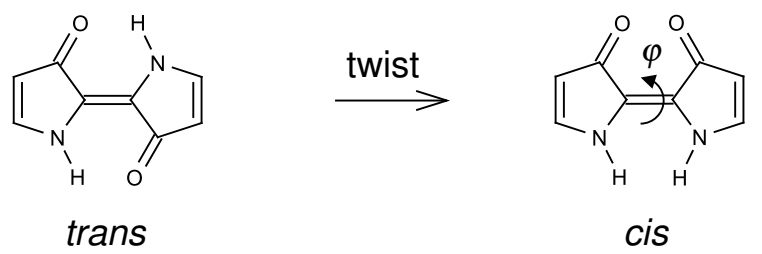
Fig. 2

(a) $S_{0}$ minimum of 1

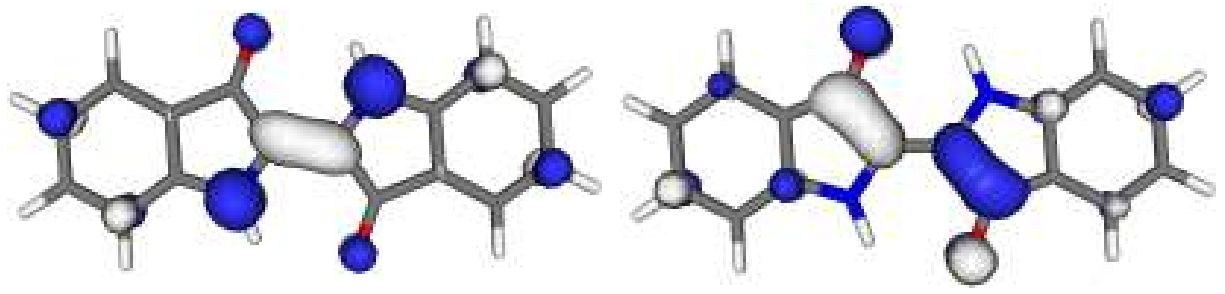

(b) $S_{0}$ minimum of 2
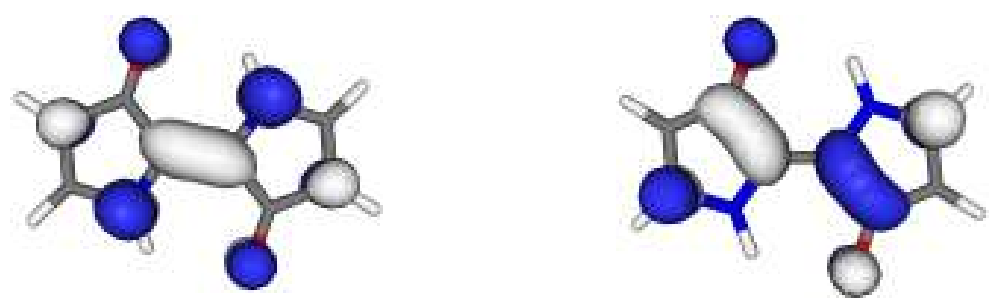
Fig. 3

(a) $\mathrm{Cl}_{\mathrm{SPT}}$
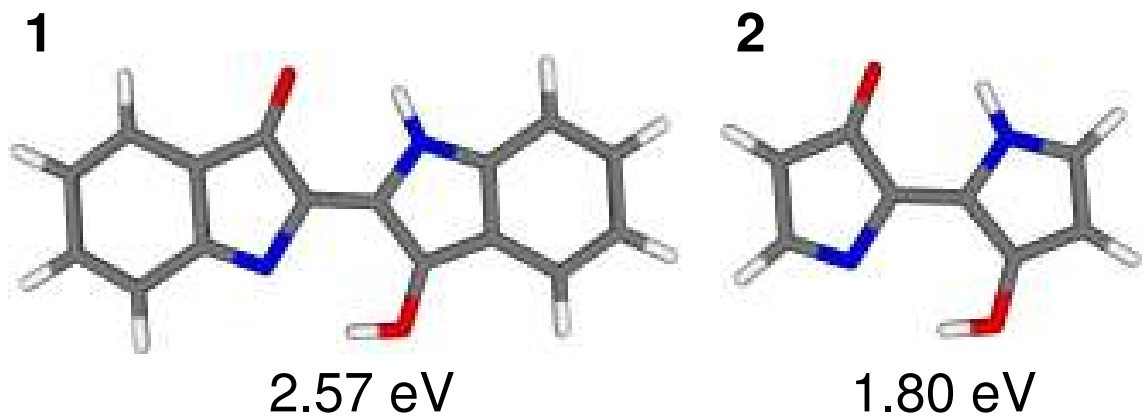

(b) $\mathrm{Cl}_{\mathrm{DPT}}$
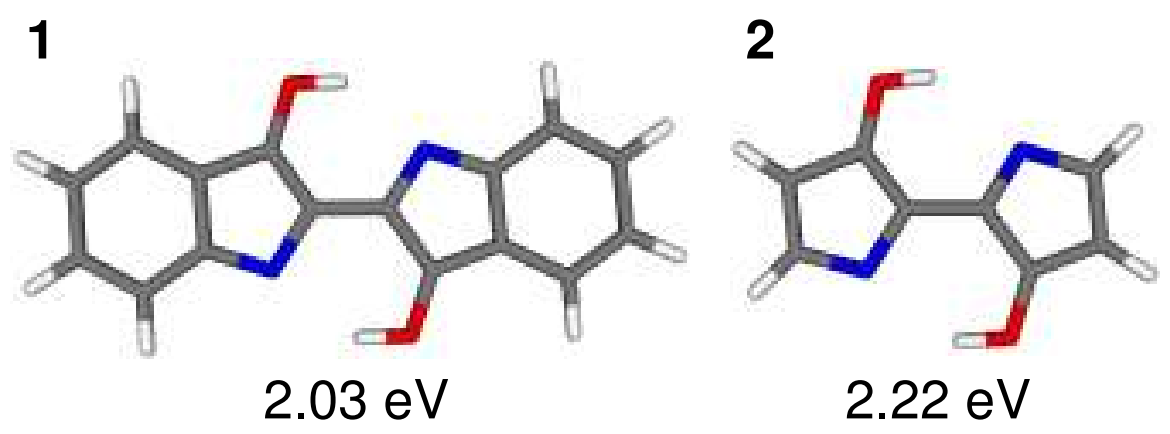

(c) $\mathrm{Cl}_{\text {twist }}$

2

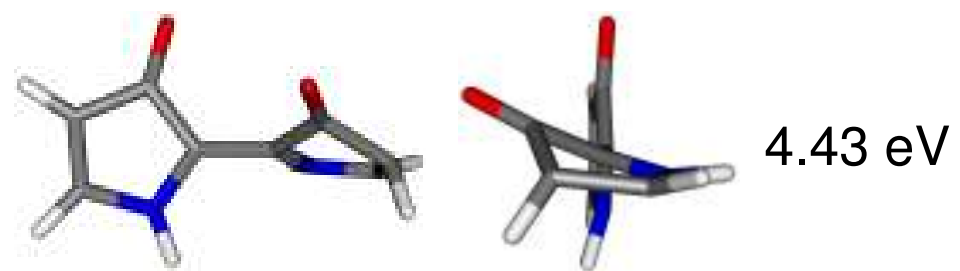


Fig. 4

(a) $\mathrm{Cl}_{\mathrm{SPT}}$ of 1

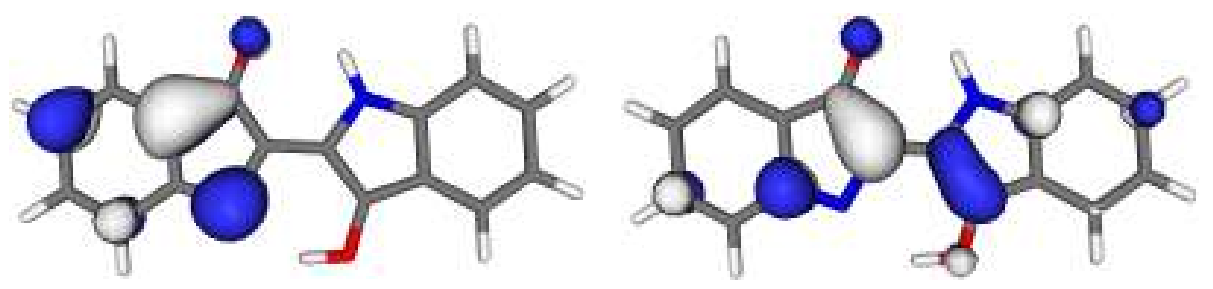

(b) $\mathrm{Cl}_{\mathrm{DPT}}$ of 1
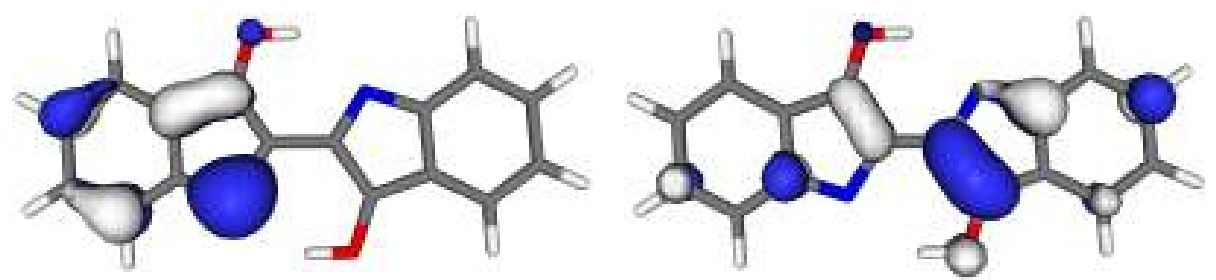

(c) $\mathrm{Cl}_{\text {twist }}$ of 2
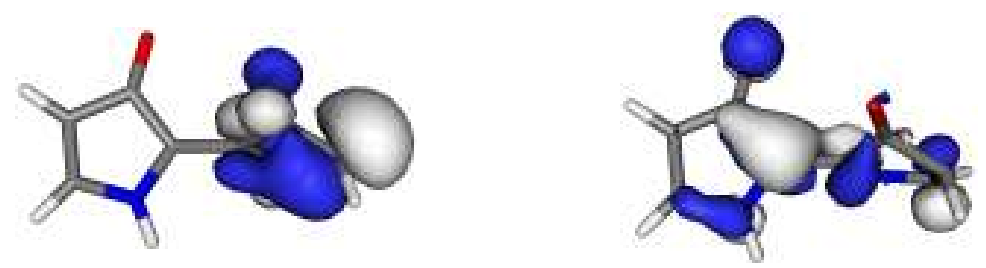
Fig. 5
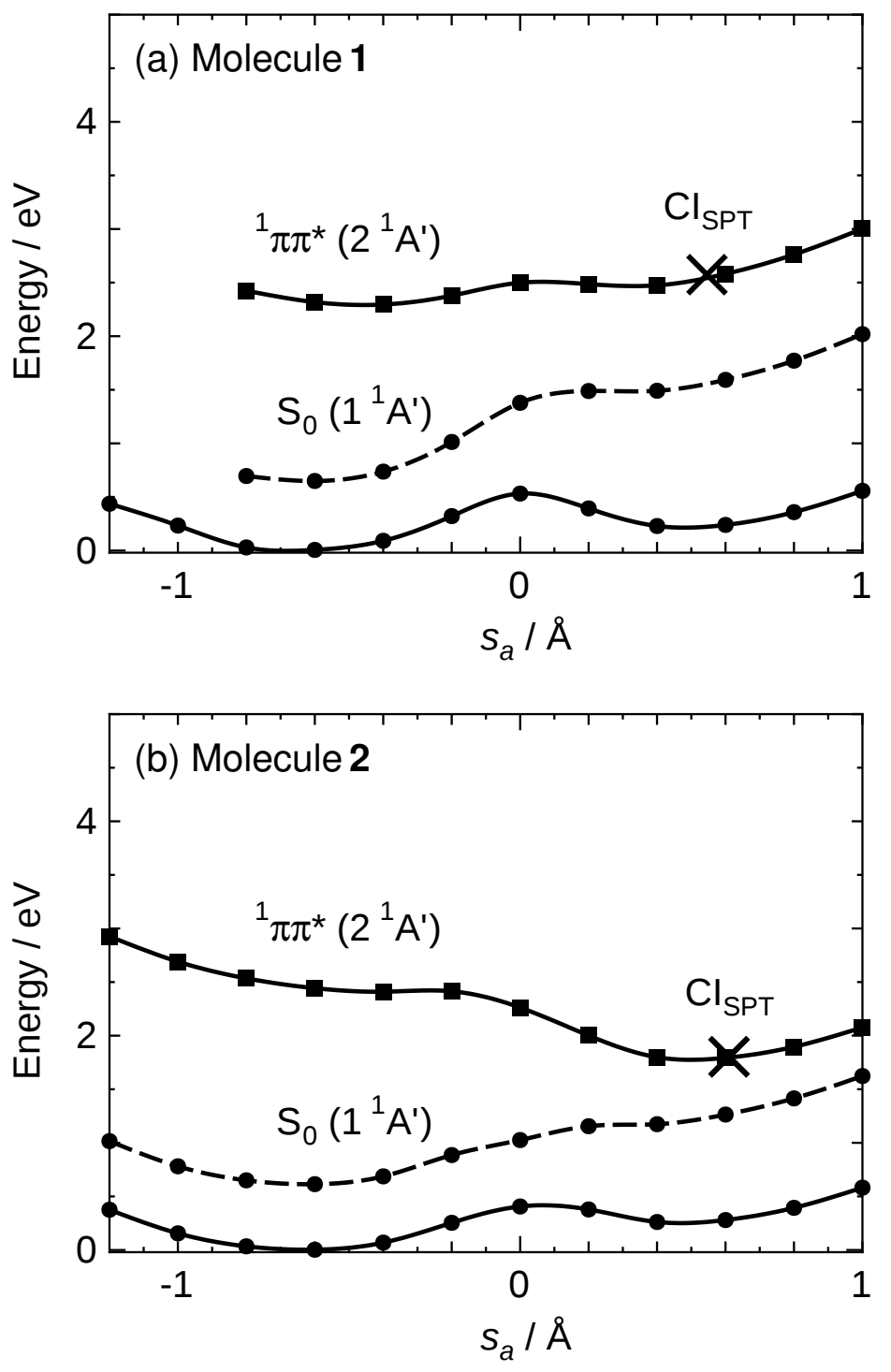
Fig. 6

(a)

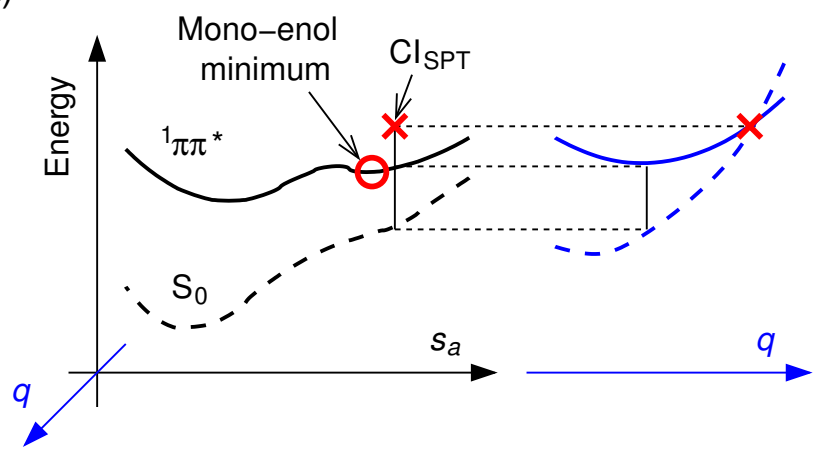

(b) Mono-enol minimum of $1\left(s_{a}=0.40 \AA\right)$

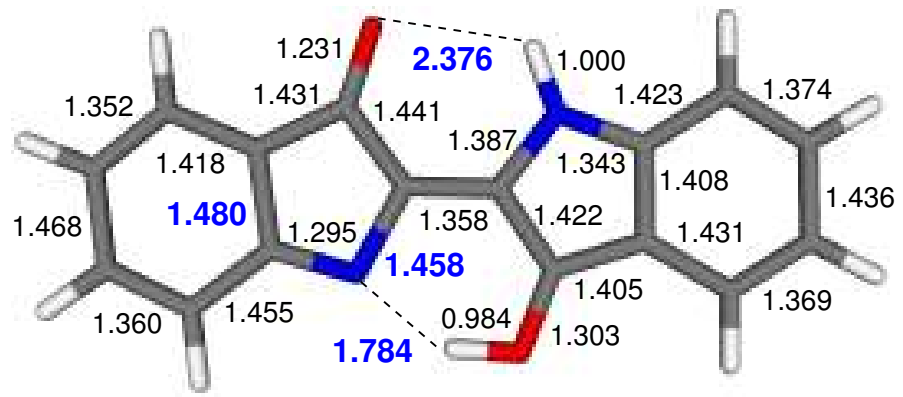

(c) $\mathrm{Cl}_{\mathrm{SPT}}$ of $1\left(s_{a}=0.55 \AA\right)$

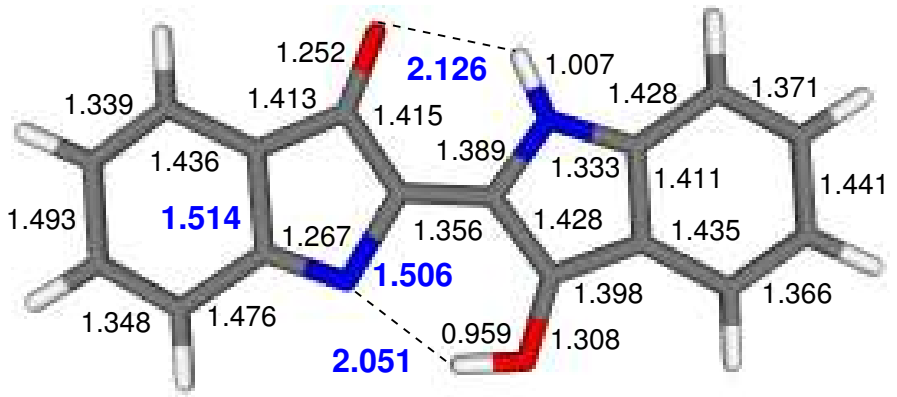


Fig. 7
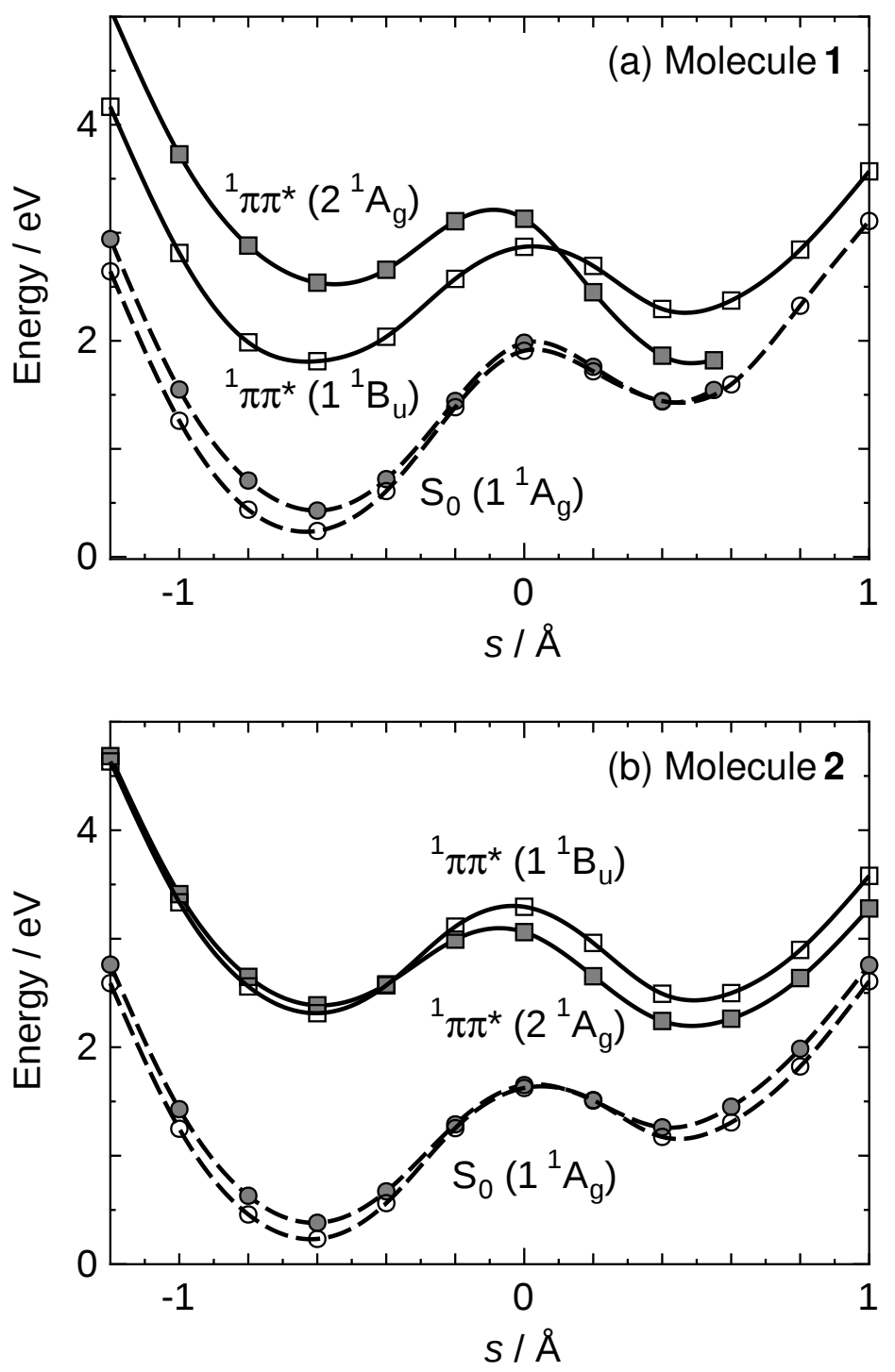
Fig. 8

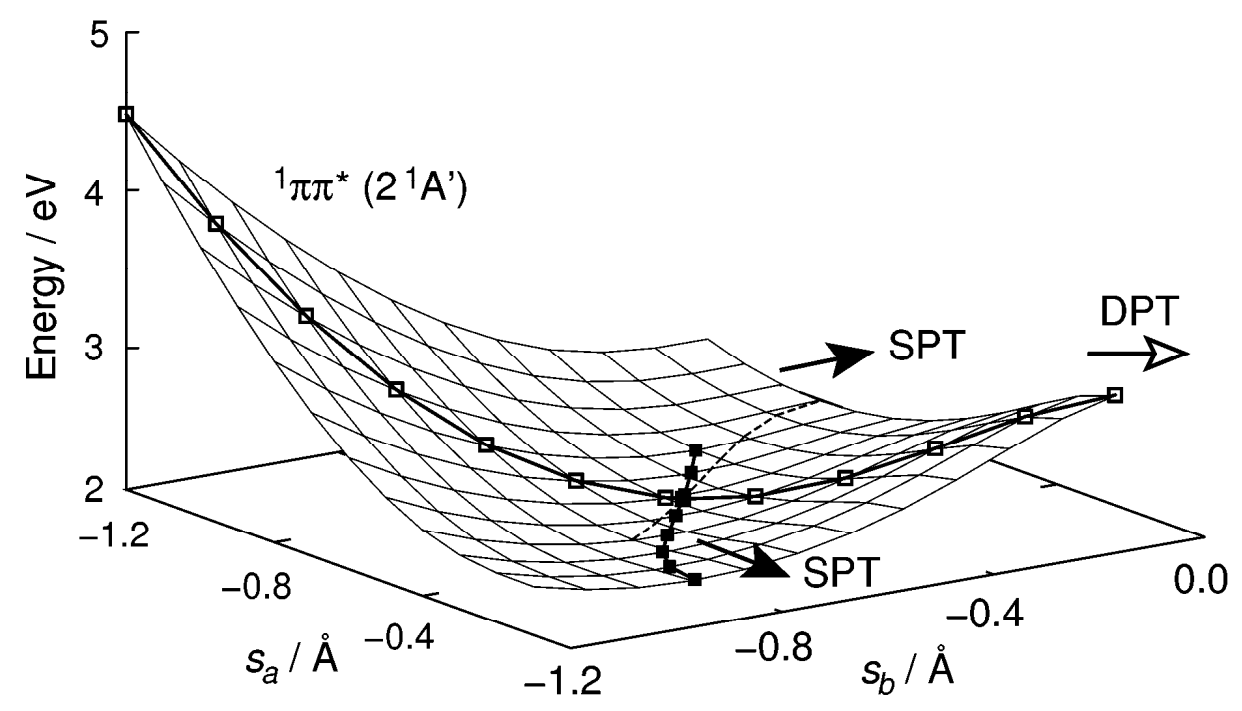


Fig. 9

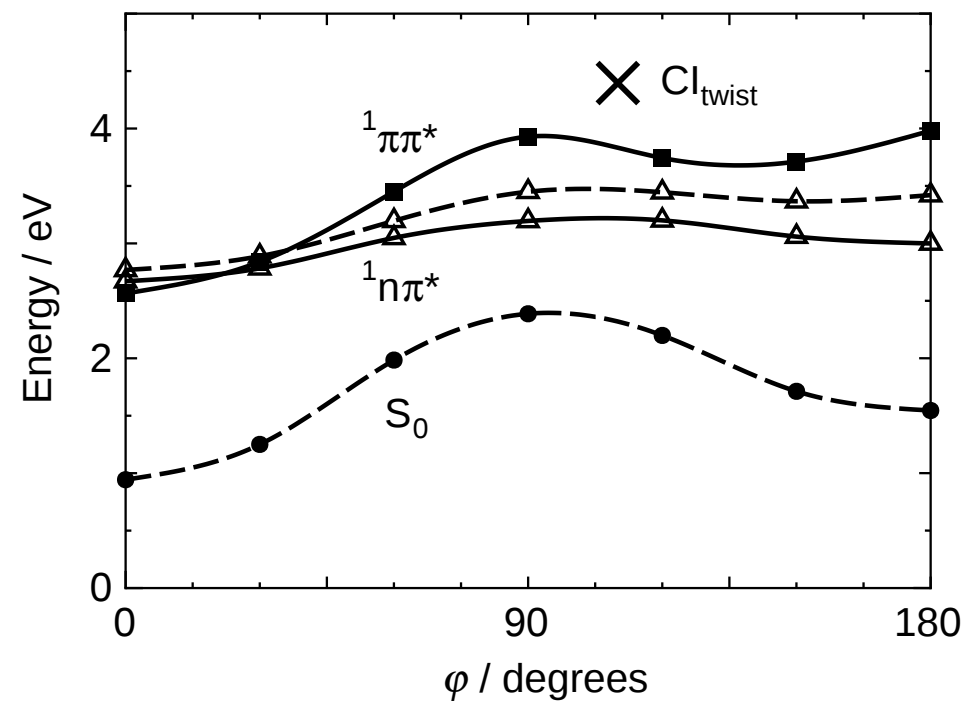


Fig. 10
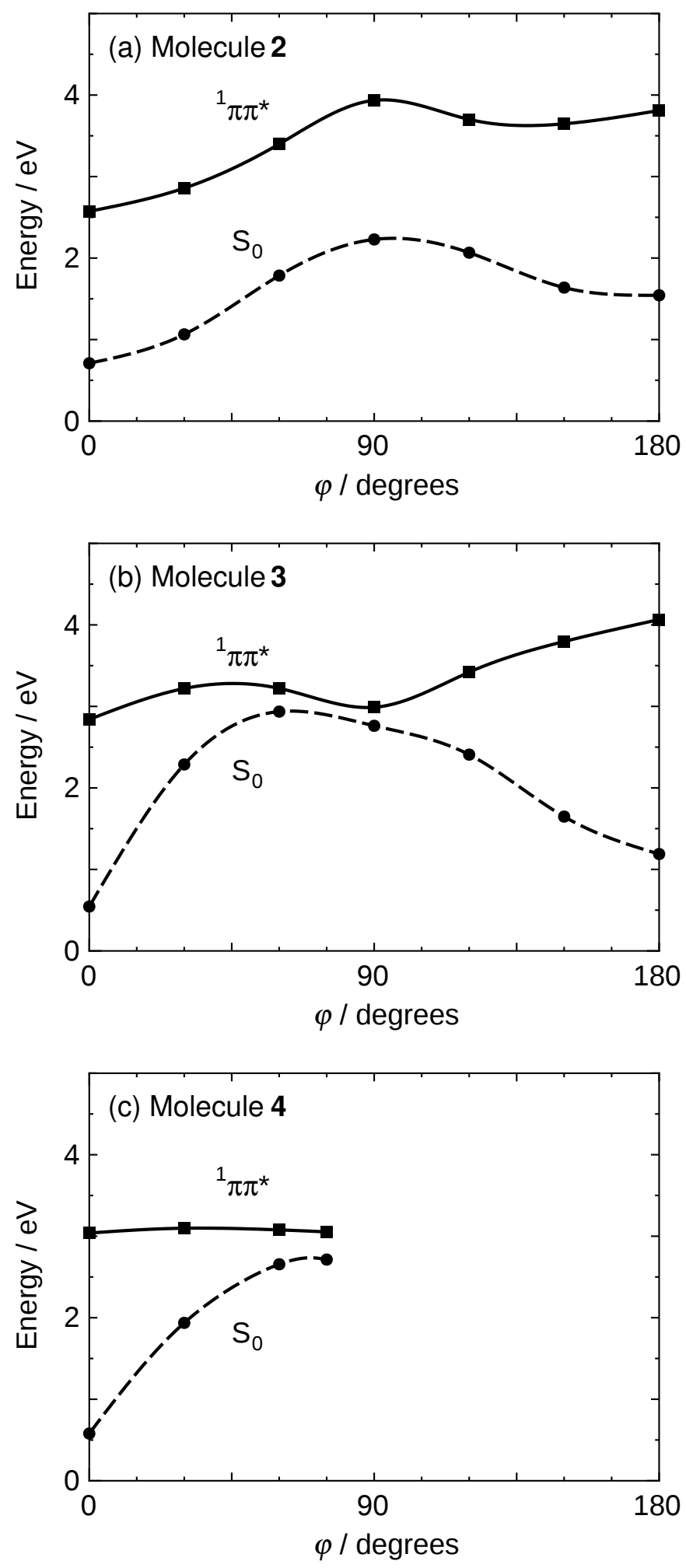
Fig. 11

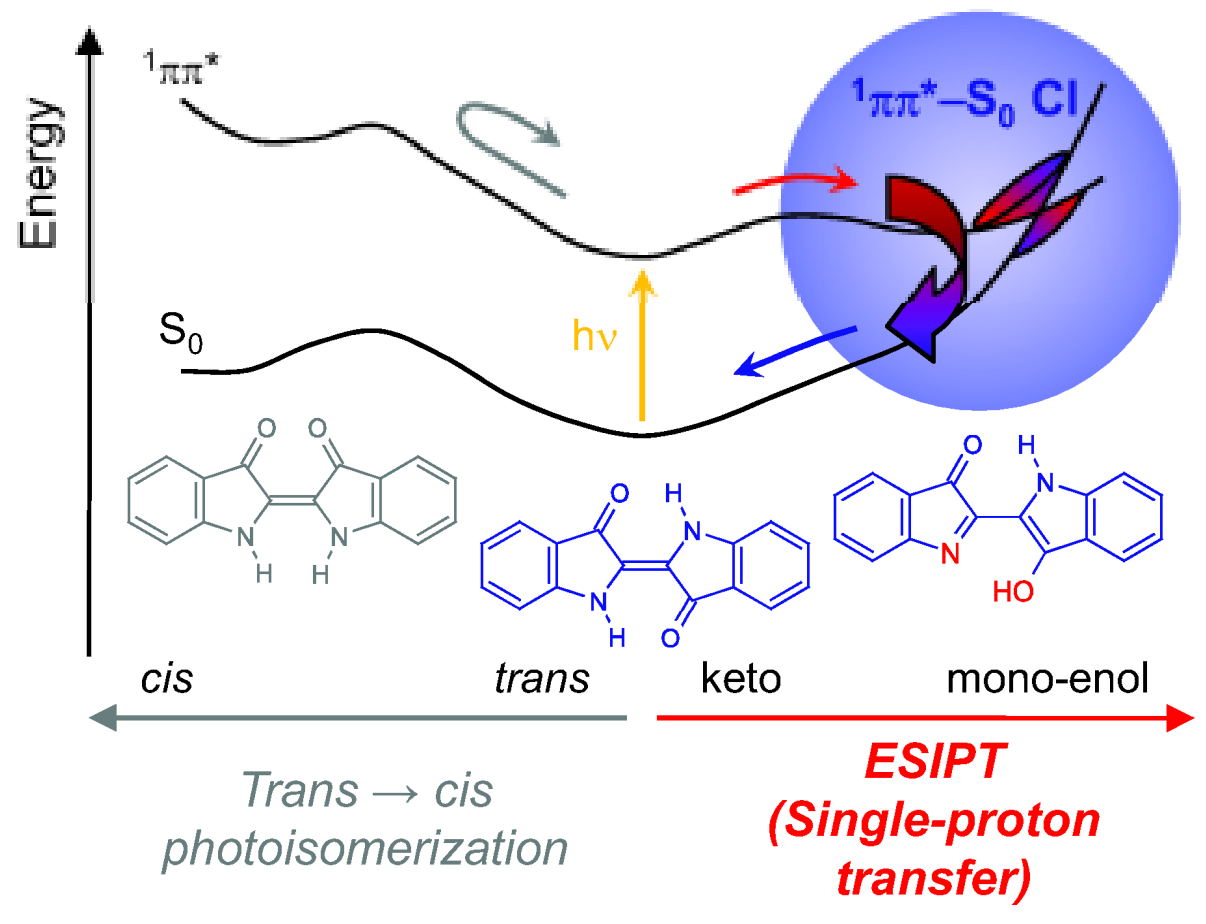

